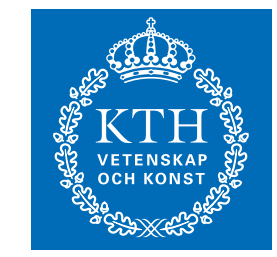

KTH Electrical Engineering

\title{
Wireless Multicast Relay Networks with Limited-Rate Source-Conferencing
}

Accepted for publication in IEEE Journal on Selected Areas in Communications, special issue on Theories and Methods for Advanced Wireless Relays. Submitted Aug. 2011, accepted May 2012. To appear on IEEE JSAC, vol. 31, no. 8, August 2013. Digital Object Identifier: 10.1109/JSAC.2013.1308.04

The manuscript was first made publicly available on this personal website in May 2012.

(C) 2012 IEEE. Personal use of this material is permitted. However, permission to reprint/republish this material for advertising or promotional purposes or for creating new collective works for resale or redistribution to servers or lists, or to reuse any copyrighted component of this work in other works must be obtained from the IEEE.

JINFENG DU, MING XIAO, MIKAEL SKOGLUND, MURIEL MÉDARD

Stockholm October 2012

School of Electrical Engineering and the ACCESS Linnaeus Center, Royal Institute of Technology (KTH), SE-100 44 Stockholm, Sweden IR-EE-KT 2012:002 


\title{
Wireless Multicast Relay Networks with Limited-Rate Source-Conferencing
}

\author{
Jinfeng Du, Student Member, IEEE, Ming Xiao, Member, IEEE, Mikael Skoglund, Senior Member, IEEE, and \\ Muriel Médard, Fellow, IEEE
}

\begin{abstract}
We investigate capacity bounds for a wireless multicast relay network where two sources simultaneously multicast to two destinations with the help of a full-duplex relay node. The two sources and the relay use the same channel resources (i.e. co-channel transmission). We assume Gaussian channels with time-invariant channel gains which are known by all nodes. The two source nodes are connected by orthogonal limited-rate errorfree conferencing links. By extending the proof of the converse for the Gaussian relay channel and introducing two lemmas on conditional (co-)variance, we present two genie-aided outer bounds of the capacity region for this multicast relay network. We extend noisy network coding to use source cooperation with the help of the theory of network equivalence. We also propose a new coding scheme, partial-decode-and-forward based linear network coding, which is essentially a hybrid scheme utilizing rate-splitting and messages conferencing at the source nodes, partial decoding and linear network coding at the relay, and joint decoding at each destination. A low-complexity alternative scheme, analog network coding based on amplify-and-forward relaying, is also investigated and shown to benefit greatly from the help of the conferencing links and can even outperform noisy network coding when the coherent combining gain is dominant.
\end{abstract}

Index Terms-Relays, source cooperation, network coding, wireless multicast, cooperative communication.

\section{INTRODUCTION}

Smart phones and tablet computers have greatly boosted the demand for services via wireless access points, keeping constant pressure on the network providers to deliver vast amounts of data over the wireless infrastructure. It becomes common that service providers may have to distribute the same content to a group of users in a small area, which makes wireless multicast an attractive option for such service delivery. As shown in Fig. 1, we consider a relay-aided twosource two-destination wireless multiple multicast network where source nodes $\mathcal{S}_{1}$ and $\mathcal{S}_{2}$ multicast their individual message $W_{1}$ at rate $R_{1}$ and $W_{2}$ at rate $R_{2}$, respectively, to both destinations $\mathcal{D}_{1}$ and $\mathcal{D}_{2}$, with the help of a relay $\mathcal{R}$. The nodes $\mathcal{S}_{1}, \mathcal{S}_{2}$, and $\mathcal{R}$ use the same channel resource

Manuscript received 30 August 2011; revised 29 February 2012.

Jinfeng Du, Ming Xiao and Mikael Skoglund are with School of Electrical Engineering and the ACCESS Linnaeus Center, Royal Institute of Technology, Stockholm, Sweden (Email: \{jinfeng, mingx, skoglund\}@kth.se).

Muriel Médard is with Research Lab of Electronics, Massachusetts Institute of Technology, Cambridge, MA, USA (Email: medard@mit.edu).

This work was funded in part by the Swedish Governmental Agency for Innovation Systems (VINNOVA) and by the Swedish Research Council (VR) project VR 621-2008-4249.

This material is also based upon work supported by the France Telecom S.A. under award No. 018499-00, and by the Air Force Office of Scientific Research (AFOSR) under award No. 016974-002.

Digital Object Identifier 10.1109/JSAC.2013.1308.04

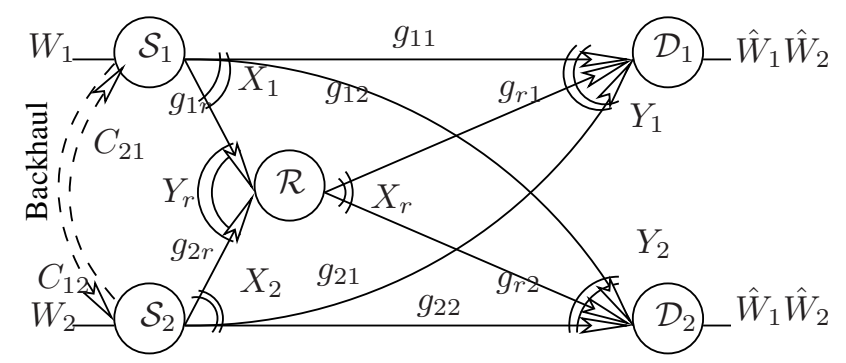

Fig. 1. Two source nodes $\mathcal{S}_{1}$ and $\mathcal{S}_{2}$, connected with backhaul (rate $C_{12}$ and $C_{12}$ ), multicast information $W_{1}$ at rate $R_{1}$ and $W_{2}$ at rate $R_{2}$ respectively to both destinations $\mathcal{D}_{1}$ and $\mathcal{D}_{2}$ through Gaussian channels, with aid from a full-duplex relay $\mathcal{R}$.

(i.e. co-channel transmission) and transmitted signals mix at all the receiving terminals and are subjected to Gaussian noise. In addition, the $\mathcal{S}_{1}$ and $\mathcal{S}_{2}$ are connected by orthogonal limited-rate error-free conferencing links (corresponding to the presence of a backhaul) with capacities $C_{12}$ and $C_{21}$, respectively. The model in Fig. 1 is generic since it covers a class of different building blocks of general wireless networks, by tuning the channel gains $g_{i j}$ and $C_{12}, C_{21}$ within the range $[0, \infty)$. It can be applied, for example, to cellular downlink scenarios where two base stations, connected through the (fiber or microwave) backhaul, multicast multimedia content to two mobile terminals, one in each cell, with the help of a dedicated relay deployed at the common cell boundary.

Significant research effort has been devoted to tackle different parts of this problem. Willems [1] introduced sourceconferencing for the discrete memoryless multiple access channel (DM-MAC) and characterized the capacity region. Bross et. al [2] extended the coding scheme to the Gaussian setting and proposed a new converse. Coding schemes and capacity regions for the compound MAC with conferencing encoders have been studied in [3], [4]. Interference channels with unidirectional conferencing encoders are investigated in [4], [5]. Capacity bounds within a constant gap for interference channels with limited source cooperation have been characterized in [6] for out-of-band source-conferencing and in [7] for in-band cooperation channels. Diversity gains by source cooperation in fading channels with full/partial channel state information (CSI) have been studied in [8][12]. The trade-off between sharing message and local CSI among source nodes through finite-rate backhaul has been studied in [13]-[15]. On the other hand, capacity results are interesting yet challenging for relay networks. Capacity bounds and various cooperative strategies have been proposed for three-node relaying networks (source-relay-sink, or two co- 
operative sources and one sink) [16], [17], for multiple-access relay channels (MARC) [18], [19] involving multiple sources and a single destination, and for broadcast relay channels (BRC) [19], [20] where a single source transmits messages to multiple destinations. Recent results on capacity bounds for multiple-source multiple-destination relay networks, [21]-[25] and references therein, have provided valuable insight into the benefits of cooperative relaying and demonstrated various tools to bound the capacity region. As different messages mix up at the relay node by nature, various network coding (NC) [26][28] approaches, which essentially combine multiple messages together, can be introduced to boost the sum rate. For instance, analog NC (ANC) with amplify-and-forward (AF) relay has been studied in [29] and proven to be asymptotically optimal [30] in multihop relay networks, linear NC and lattice codes with decode-and-forward relay are investigated in [24]. The recently proposed noisy NC scheme [31] enables multiple multicasts over noisy networks without explicit decoding at intermediate nodes.

In our previous work [32], [33], we combined source cooperation and network coding in multicast relay networks. For the scenario when the source nodes can fully cooperate, i.e., the conferencing rate is high $\left(C_{12} \geq R_{1}, C_{21} \geq R_{2}\right)$, we presented the exact cut-set bound and proposed several cooperative NC strategies. The goal of the present paper is to gain deeper understanding of such systems in a more realistic setting and demonstrate the benefit of combining source cooperation with relaying. In this work, we therefore focus on the limited conferencing $\left(0 \leq C_{12}<R_{1}, 0 \leq C_{21}<R_{2}\right)$ scenario and the results to be presented here are hence more general since they recover our previous results by simply increasing the conferencing rate. More precisely, we have developed a new way to upper bound the performance by introducing a genie and two lemmas on conditional (co-)variance, which help us to find two outer bounds following a similar procedure as in [32], [33]. We also investigate three achievable rate strategies where the relay may decode, compress, or simply amplify the received signals, respectively. Based on network equivalence [34], we extend the noisy $\mathrm{NC}$ scheme to use the conferencing links. We explain the key steps in computation of its rate regions and point out its limitations on maximizing the sum-rate. Motivated by the result that sending common messages from both source nodes can achieve capacity under the conditions specified in [32], we propose a partial-decode-andforward based linear network coding ( $\mathrm{pDF}+\mathrm{LNC})$ scheme: $\mathcal{S}_{1}$ and $\mathcal{S}_{2}$ perform message-splitting and then exchange messages via conferencing links prior to each transmission; $\mathcal{R}$ decodes part of the received messages and forward a combination of them via linear network coding; $\mathcal{D}_{1}$ and $\mathcal{D}_{2}$ perform joint decoding. ANC based on AF relaying is also investigated as a low-complexity alternative and shown to be very effective when source cooperation is possible.

The remaining part of this paper is organized as follows. Sec. II introduces the system model and Sec. III presents two outer bounds. The extension of the noisy NC scheme is described in Sec. IV. Sec. V characterizes the achievable rate regions for $\mathrm{pDF}+\mathrm{LNC}$ as well as ANC. Sec. VI presents the numerical illustrations and concluding remarks are in Sec. VII.

\section{List of Notation}

- $X$ (and $Y, Z, U, V$ ): real valued random variable (with $x$ as a realization)

- $X^{(n)}$ : a vector of $X$ of length $n$ (indicate a codeword or a sequence of symbols/signals)

- $p(x)$ : probability density/mass function of $X$

- $h(X)$ : differential entropy of $X$

- $I(X ; Y)$ : mutual information between $X$ and $Y$

- $\alpha, \rho \in[0,1]:$ auxiliary random variables reserved as power allocation parameters

- $\mathcal{N}\left(\mu, \sigma^{2}\right)$ : Gaussian distribution with mean $\mu$ and variance $\sigma^{2}$

- $\mathcal{C}(x)=\frac{1}{2} \log (1+x)$ : Gaussian capacity function.

\section{System Model}

We assume all the individual channel gains $g_{i j} \geq 0$, $i, j=1,2, r$ are time-invariant and known to every node in the network. The scenario of only local/partial CSI, requiring a trade-off between message and CSI exchange as demonstrated in [13]-[15], is left to future work. Given an average transmit power constraint $P$, fixed channel gain $g$, and noise power $N$, the signal-to-noise ratio (SNR) of an individual link can be written as $\gamma=g^{2} P / N$. We can therefore characterize the transmission links by only their individual SNR $\gamma$, without distinguishing the SNR contribution. The system shown in Fig. 1 can be modelled as follows

$$
\begin{array}{rr}
Y_{1}^{(n)}=\sqrt{\gamma_{11}} X_{1}^{(n)}+\sqrt{\gamma_{21}} X_{2}^{(n)} & +\sqrt{\gamma_{r 1}} X_{r}^{(n)}+Z_{1}^{(n)}, \\
Y_{2}^{(n)}=\sqrt{\gamma_{12}} X_{1}^{(n)}+\sqrt{\gamma_{22}} X_{2}^{(n)} & +\sqrt{\gamma_{r 2}} X_{r}^{(n)}+Z_{2}^{(n)}, \\
Y_{r}^{(n)}=\sqrt{\gamma_{1 r}} X_{1}^{(n)}+\sqrt{\gamma_{2 r}} X_{2}^{(n)} & +Z_{r}^{(n)},
\end{array}
$$

where $\gamma_{i j} \geq 0, i, j=1,2, r$ are the effective link SNR, $X_{i}^{(n)}$, $Y_{i}^{(n)}, Z_{i}^{(n)}, i=1,2, r$ are $n$-dimensional transmitted signals, received signals, and additive noise, respectively. Noise components $Z_{i, k}, i=1,2, r$ and $k=1, \ldots, n$ are i.i.d. Gaussian with zero-mean unit-variance. All the transmitted signals are subject to average unit-power constraints, i.e.,

$$
\frac{1}{n} \sum_{k=1}^{n} X_{i, k}^{2} \leq 1
$$

\section{Genie-Aided Outer Bounds}

\section{A. The Cut-Set Bound}

By the cut-set bound [35], the maximum achievable rate from the source nodes to any of the destinations can be no larger than the minimum of the mutual information flows across all possible cuts, maximized over a joint distribution for the transmitted signals.

Proposition 1: The cut-set bound for the multicast network in Fig. 1 can be characterized by

$$
\begin{aligned}
& C_{\text {cut-set }}=\bigcup_{p\left(x_{1}, x_{2}, x_{r}\right)}\{\left(R_{1}, R_{2}\right): R_{1} \geq 0, R_{2} \geq 0, \\
& R_{1} \leq C_{12}+\frac{1}{n} \min _{d \in\{1,2\}}\left\{I\left(X_{1}^{(n)} X_{r}^{(n)} ; Y_{d}^{(n)} \mid X_{2}^{(n)} X_{s}^{(n)}\right),\right. \\
&\left.I\left(X_{1}^{(n)} ; Y_{d}^{(n)} Y_{r}^{(n)} \mid X_{2}^{(n)} X_{r}^{(n)} X_{s}^{(n)}\right)\right\}+\epsilon_{n}, \\
& R_{2} \leq C_{21}+\frac{1}{n} \min _{d \in}\{1,2\}\left\{I\left(X_{2}^{(n)} X_{r}^{(n)} ; Y_{d}^{(n)} \mid X_{1}^{(n)} X_{s}^{(n)}\right),\right. \\
& \\
&\left.I\left(X_{2}^{(n)} ; Y_{d}^{(n)} Y_{r}^{(n)} \mid X_{1}^{(n)} X_{r}^{(n)} X_{s}^{(n)}\right)\right\}+\epsilon_{n},
\end{aligned}
$$




$$
\begin{array}{r}
R_{1}+R_{2} \leq \frac{1}{n} \min _{d \in\{1,2\}}\left\{I\left(X_{1}^{(n)} X_{2}^{(n)} X_{r}^{(n)} ; Y_{d}^{(n)}\right),\right. \\
\left.I\left(X_{1}^{(n)} X_{2}^{(n)} ; Y_{d}^{(n)} Y_{r}^{(n)} \mid X_{r}^{(n)}\right)\right\}+\epsilon_{n}, \\
R_{1}+R_{2} \leq C_{12}+C_{21}+\frac{1}{n} \min _{d \in\{1,2\}}\left\{I\left(X_{1}^{(n)} X_{2}^{(n)} X_{r}^{(n)} ; Y_{d}^{(n)} \mid X_{s}^{(n)}\right),\right. \\
\left.\left.I\left(X_{1}^{(n)} X_{2}^{(n)} ; Y_{d}^{(n)} Y_{r}^{(n)} \mid X_{r}^{(n)} X_{s}^{(n)}\right)\right\}+\epsilon_{n}\right\}
\end{array}
$$

where $X_{s}^{(n)}$ represent symbols transmitted via the conferencing links, $X_{1}, X_{2}$ and $X_{r}$ are subject to the average power constraint (2), $\epsilon_{n} \rightarrow 0$ as $n \rightarrow \infty$, and the joint probability is partitioned as $p\left(x_{s}, x_{r}\right) p\left(x_{1} \mid x_{s}, x_{r}\right) p\left(x_{2} \mid x_{s}, x_{r}\right) p\left(y_{r} \mid x_{1}, x_{2}\right)$ $\times p\left(y_{1} \mid x_{1}, x_{2}, x_{r}\right) p\left(y_{2} \mid x_{1}, x_{2}, x_{r}\right)$.

Proof: Follows directly from [35] by evaluating all the possible cuts and from [1] by taking into account the power constraint and the correlation between $X_{1}, X_{2}$ and $X_{r}$.

\section{B. Genie-Aided Outer Bound}

By extending the proof of the converse developed by Cover and El Gamal [16] for the Gaussian relay channel, we have characterized the exact cut-set bound for a multicast relay network supported by a high-rate backhaul (i.e., $C_{12} \geq R_{1}$ and $C_{21} \geq R_{2}$ ) with/without cross-links [32], [33]. However, it is difficult to directly apply that result here since the transmitted signal at the relay is only partially known to both source nodes owing to the limited-rate conferencing links. Instead, we introduce a genie which tells the two source nodes exactly what the relay is going to transmit, i.e., $X_{r}$ is known at $\mathcal{S}_{1}$ and $\mathcal{S}_{2}$ non-causally. Therefore $X_{r}$ needs not to be transmitted via the conferencing links, i.e., the conferencing symbols $X_{s}^{(n)}$ are independent of $X_{r}^{(n)}$, which indicates that $p\left(x_{r}, x_{s}\right)=p\left(x_{r}\right) p\left(x_{s}\right)$ is sufficient for the probability partition in Proposition 1. Since $X_{1}$ is potentially correlated to $X_{r}$ and $X_{s}$, we can introduce two independent auxiliary variables $\alpha_{1}, \rho_{1} \in[0,1]$ to indicate the dependence of $X_{1}$ on $X_{r}$ (via $\bar{\alpha}_{1}=1-\alpha_{1}$ ) and on $X_{s}$ (via $\rho_{1} \alpha_{1}$ ). Similarly, $\alpha_{2}, \rho_{2} \in[0,1]$ are introduced for $X_{2}$. Following similar procedures as in [32], [33], we can bound all the mutual information terms in (3) and obtain the following outer bound.

Proposition 2: The cut-set bound $C_{\text {cut-set }}$ in Proposition 1 can be outer bounded by

$$
\begin{gathered}
C_{\text {upp } 1}=\bigcup_{0 \leq \alpha_{1}, \alpha_{2}, \rho_{1}, \rho_{2} \leq 1}\left\{\left(R_{1}, R_{2}\right): R_{1} \geq 0, R_{2} \geq 0,\right. \\
R_{1} \leq C_{12}+\min _{d \in\{1,2\}}\left\{\mathcal{C}\left(\left(\gamma_{1 d}+\gamma_{1 r}\right) \bar{\rho}_{1} \alpha_{1}\right),\right. \\
\left.\mathcal{C}\left(\gamma_{1 d}\left(\bar{\rho}_{1} \alpha_{1}+\bar{\alpha}_{1}\right)+\gamma_{r d}+2 \sqrt{\gamma_{1 d} \gamma_{r d} \bar{\alpha}_{1}}\right)\right\} \\
R_{2} \leq C_{21}+\min _{d \in\{1,2\}}\left\{\mathcal{C}\left(\left(\gamma_{2 d}+\gamma_{2 r}\right) \bar{\rho}_{2} \alpha_{2}\right),\right. \\
\left.\mathcal{C}\left(\gamma_{2 d}\left(\bar{\rho}_{2} \alpha_{2}+\bar{\alpha}_{2}\right)+\gamma_{r d}+2 \sqrt{\gamma_{2 d} \gamma_{r d} \bar{\alpha}_{2}}\right)\right\} \\
R_{1}+R_{2} \leq \min _{d \in\{1,2\}}\left\{\mathcal { C } \left(\gamma_{1 d}+\gamma_{2 d}+\gamma_{r d}+2 \sqrt{\bar{\alpha}_{1} \gamma_{1 d} \gamma_{r d}}\right.\right. \\
\left.+2 \sqrt{\bar{\alpha}_{2} \gamma_{2 d} \gamma_{r d}}+2 \sqrt{\gamma_{1 d} \gamma_{2 d}}\left(\sqrt{\rho_{1} \rho_{2} \alpha_{1} \alpha_{2}}+\sqrt{\bar{\alpha}_{1} \bar{\alpha}_{2}}\right)\right), \\
\mathcal{C}\left(\left(\gamma_{1 d}+\gamma_{1 r}\right) \alpha_{1}+\left(\sqrt{\gamma_{1 d} \gamma_{2 r}}-\sqrt{\gamma_{2 d} \gamma_{1 r}}\right)^{2} \alpha_{1} \alpha_{2}\left(1-\lambda_{d}^{2} \rho_{1} \rho_{2}\right)\right. \\
\left.\left.+\left(\gamma_{2 d}+\gamma_{2 r}\right) \alpha_{2}+2\left(\sqrt{\gamma_{1 d} \gamma_{2 d}}+\sqrt{\gamma_{1 r} \gamma_{2 r}}\right) \lambda_{d} \sqrt{\rho_{1} \rho_{2} \alpha_{1} \alpha_{2}}\right)\right\} \\
R_{1}+R_{2} \leq C_{12}+C_{21}+\min _{d \in\{1,2\}}\left\{\mathcal { C } \left(\left(\gamma_{1 d}+\gamma_{1 r}\right) \bar{\rho}_{1} \alpha_{1}\right.\right. \\
\left.+\left(\gamma_{2 d}+\gamma_{2 r}\right) \bar{\rho}_{2} \alpha_{2}+\left(\sqrt{\gamma_{1 d} \gamma_{2 r}}-\sqrt{\gamma_{2 d} \gamma_{1 r}}\right)^{2} \bar{\rho}_{1} \bar{\rho}_{2} \alpha_{1} \alpha_{2}\right), \\
\mathcal{C}\left(\gamma_{1 d}\left(\bar{\alpha}_{1}+\bar{\rho}_{1} \alpha_{1}\right)+\gamma_{2 d}\left(\bar{\alpha}_{2}+\bar{\rho}_{2} \alpha_{2}\right)+\gamma_{r d}\right. \\
\left.\left.\left.+2 \sqrt{\gamma_{1 d} \gamma_{2 d} \bar{\alpha}_{1} \bar{\alpha}_{2}}+2 \sqrt{\gamma_{1 d} \gamma_{r d} \bar{\alpha}_{1}}+2 \sqrt{\gamma_{2 d} \gamma_{r d} \bar{\alpha}_{2}}\right)\right\}\right\}
\end{gathered}
$$

with $\bar{\alpha}_{1}=1-\alpha_{1}, \bar{\alpha}_{2}=1-\alpha_{2}, \bar{\rho}_{1}=1-\rho_{1}, \rho^{2} \leq \rho_{1}$, and $\rho^{2} / \rho_{1}=$ 0 for $\rho=\rho_{1}=0$.

Proof: The proof can be found in Appendix B.

Proposition 4: Let $C_{\mathrm{upp} 3}$ be the region obtained directly from (5) by variable substitution $\left(\rho^{2} / \rho_{1}\right.$ is treated as a single variable) as follows: $\rho^{2} / \rho_{1} \Leftrightarrow \rho_{2}, 1-\rho^{2} / \rho_{1} \Leftrightarrow \bar{\rho}_{2}$ and $\rho_{1} \Leftrightarrow \rho^{2} / \rho_{2}, \quad \bar{\rho}_{1} \Leftrightarrow 1-\rho^{2} / \rho_{2}$. We can outer bound $C_{\text {cut-set }}$ by $C_{\text {upp } 4}=C_{\text {upp } 2} \cap C_{\text {upp } 3}$.

Proof: It is sufficient to prove $C_{\text {cut-set }} \subseteq C_{\text {upp3 }}$ by following the same procedure as in Appendix B except introducing $\rho_{2}$ (instead of $\rho_{1}$ ) such that

$$
\bar{\rho}_{2} \alpha_{2}=\frac{1}{n} \sum_{i=1}^{n} E\left[\operatorname{Var}\left(X_{2, i} \mid X_{r, i} X_{s, i}\right)\right] .
$$

The supremum operation should be over $0 \leq \alpha_{1}, \alpha_{2}, \rho, \rho_{2} \leq 1$ accordingly with $\rho^{2} \leq \rho_{2}$. 


\section{Noisy Network Coding with Source COOPERATION}

In this section, we provide an inner bound of the capacity region by an extension of the noisy NC scheme. The basic principle of noisy $\mathrm{NC}$, as described in [31], is to convey a "super message" $B$ times, each time using an independent codebook and letting $B \rightarrow \infty$, before the destination(s) can successfully decode the message. Therefore collaboration by sharing messages via conferencing bit-pipes is not feasible since it requires a $B \rightarrow \infty$ times higher conferencing rate to exchange the super message before transmission starts. On the other hand, the orthogonal conferencing bit-pipes between two source nodes can serve as relay nodes for each other. According to the theory of network equivalence [34], the capacity of a network is unchanged if any independent, memoryless, pointto-point channel in this network is replaced by a noiseless bitpipe with throughput equal to the removed channel's capacity. Since the conferencing bit-pipes between two source nodes are independent and orthogonal to all the other transmissions, they can be replaced [34] by noisy channels with the same capacity as follows:

$$
\begin{aligned}
& C_{12}: Y_{s 2}=\sqrt{P_{1}} X_{s 1}+Z_{s 2}, \text { with } \mathcal{C}\left(P_{1}\right)=C_{12}, \\
& C_{21}: Y_{s 1}=\sqrt{P_{2}} X_{s 2}+Z_{s 1}, \text { with } \mathcal{C}\left(P_{2}\right)=C_{21},
\end{aligned}
$$

where $X_{s 1}, X_{s 2}, Z_{s 1}, Z_{s 2}$ are independent Gaussian ${ }^{1}$ random variables with zero-mean and unit-variance, $P_{1}, P_{2}$ are corresponding power constraints, and $Y_{s 1}, Y_{s 2}$ are the conferencing outputs at source nodes $\mathcal{S}_{1}$ and $\mathcal{S}_{2}$, respectively. Note that signals in (6) are orthogonal to all the other transmissions and therefore will not mix with signals (e.g. $X_{1}, X_{2}$ ) in (1). Now we can extend the noisy NC scheme [31], originally designed for co-channel relay networks, to our setup with orthogonal conferencing bit-pipes.

Proposition 5: An achievable rate region of noisy NC with conferencing encoders is obtained as the union of all rate pairs $\left(R_{1}, R_{2}\right)$ that satisfy $R_{1} \geq 0, R_{2} \geq 0$, and

$$
\begin{aligned}
R_{1}<\Delta_{R_{1}}+\min \left\{\mathcal{C}\left(\gamma_{11}+\frac{\gamma_{1 r}}{1+\sigma_{r}^{2}}\right), \mathcal{C}\left(\gamma_{12}+\frac{\gamma_{1 r}}{1+\sigma_{r}^{2}}\right),\right. \\
\left.\mathcal{C}\left(\gamma_{11}+\gamma_{r 1}\right)-\mathcal{C}\left(1 / \sigma_{r}^{2}\right), \mathcal{C}\left(\gamma_{12}+\gamma_{r 2}\right)-\mathcal{C}\left(1 / \sigma_{r}^{2}\right)\right\}, \\
R_{2}<\Delta_{R_{2}}+\min \left\{\mathcal{C}\left(\gamma_{21}+\frac{\gamma_{2 r}}{1+\sigma_{r}^{2}}\right), \mathcal{C}\left(\gamma_{22}+\frac{\gamma_{2 r}}{1+\sigma_{r}^{2}}\right),\right. \\
\left.\mathcal{C}\left(\gamma_{21}+\gamma_{r 1}\right)-\mathcal{C}\left(1 / \sigma_{r}^{2}\right), \mathcal{C}\left(\gamma_{22}+\gamma_{r 2}\right)-\mathcal{C}\left(1 / \sigma_{r}^{2}\right)\right\}, \\
R_{1}+R_{2}<\Delta_{R_{s}}+\min \left\{\mathcal{C}\left(\gamma_{11}+\gamma_{21}+\gamma_{r 1}\right)-\mathcal{C}\left(1 / \sigma_{r}^{2}\right),\right. \\
\mathcal{C}\left(\gamma_{12}+\gamma_{22}+\gamma_{r 2}\right)-\mathcal{C}\left(1 / \sigma_{r}^{2}\right), \\
\mathcal{C}\left(\gamma_{11}+\gamma_{21}+\frac{\gamma_{1 r}+\gamma_{2 r}+\left(\sqrt{\gamma_{11} \gamma_{2 r}}-\sqrt{\gamma_{21} \gamma_{1 r}}\right)^{2}}{1+\sigma_{r}^{2}}\right), \\
\left.\mathcal{C}\left(\gamma_{12}+\gamma_{22}+\frac{\gamma_{1 r}+\gamma_{2 r}+\left(\sqrt{\gamma_{12} \gamma_{2 r}}-\sqrt{\gamma_{22} \gamma_{1 r}}\right)^{2}}{1+\sigma_{r}^{2}}\right)\right\},
\end{aligned}
$$

where $\Delta_{R_{1}}=\mathcal{C}\left(\frac{P_{1}}{1+\sigma_{2}^{2}}\right)-\mathcal{C}\left(\frac{1}{\sigma_{1}^{2}}\right), \quad \Delta_{R_{2}}=\mathcal{C}\left(\frac{P_{2}}{1+\sigma_{1}^{2}}\right)-\mathcal{C}\left(\frac{1}{\sigma_{2}^{2}}\right)$, and $\Delta_{R_{s}}=-\mathcal{C}\left(1 / \sigma_{1}^{2}\right)-\mathcal{C}\left(1 / \sigma_{2}^{2}\right)$, and the union is taken over all $\sigma_{1}^{2}, \sigma_{2}^{2}, \sigma_{r}^{2}>0$. The value of $P_{1}, P_{2}$ is determined by conferencing rate $\left(C_{12}, C_{21}\right)$ as defined in (6).

\footnotetext{
${ }^{1}$ In [34] the noisy channel is only required to have the same capacity as the bit-pipe's throughput, with no restriction on the channel input or output. By restricting ourselves to Gaussian signals, the capacity of the overall network will not be increased, and therefore we still have a valid capacity inner bound.
}

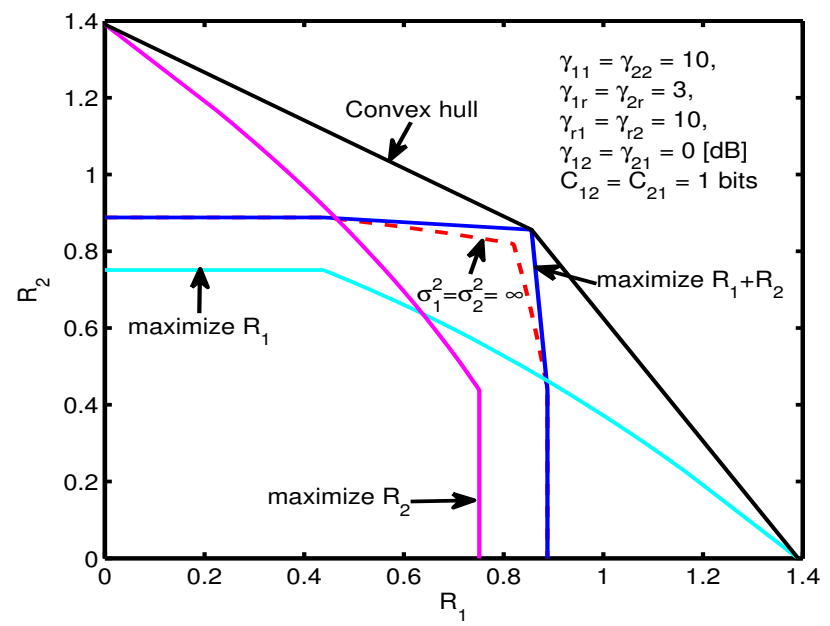

Fig. 2. Achievable rate region of Noisy NC with conferencing links, achieved by time-sharing among rate optimization of $R_{1}, R_{2}$, and $R_{1}+R_{2}$, respectively. The SNR parameters are heuristically chosen.

Remark 1: $\sigma_{i}^{2}, i=1,2, r$, refers to the controllable quantization noise power induced by noisy compression at $\mathcal{S}_{1}, \mathcal{S}_{2}$, and $\mathcal{R}$, respectively, which leads to a rate penalty $-\mathcal{C}\left(1 / \sigma_{i}^{2}\right)$. Rate contributions $\mathcal{C}\left(\frac{P_{1}}{1+\sigma_{2}^{2}}\right)$ and $\mathcal{C}\left(\frac{P_{2}}{1+\sigma_{1}^{2}}\right)$ are due to noisy relaying of the conferencing messages. Since $\Delta_{R_{s}} \leq 0$ with equality if and only if $\sigma_{1}^{2}=\sigma_{2}^{2}=\infty$, i.e., no source cooperation via conferencing links, we have to compute the rate region for noisy $\mathrm{NC}$ in three steps: first generate the rate region of noisy NC without utilizing conferencing links; then compute rate regions by maximizing $R_{1}, R_{2}$, and $R_{1}+R_{2}$, respectively; finally, apply time-sharing among different optimization schemes to get the rate region, as illustrated in Fig. 2. The maximization of $R_{1}+R_{2}$ is not always necessary. For example, if $0<C_{12}, C_{21} \leq \frac{1}{2}$, we have $P_{1} \leq 1$ and $P_{2} \leq 1$ according to (6). Then for any $0<\sigma_{1}^{2}, \sigma_{2}^{2}<\infty$ we have $\Delta_{R_{1}}+\Delta_{R_{2}}<0$ and $\Delta_{R_{s}}<0$, i.e., the sum-rate $R_{1}+R_{2}$ cannot be increased.

Proof: Given the set of transmitting nodes $T=$ $\left\{\mathcal{S}_{1}, \mathcal{S}_{2}, \mathcal{R}\right\}$ and the set of sink nodes $D=\left\{\mathcal{D}_{1}, \mathcal{D}_{2}\right\}$, and denoting $R_{1}=R\left(\mathcal{S}_{1}\right), R_{2}=R\left(\mathcal{S}_{2}\right), R(\mathcal{R})=0$, the achievable rate region of noisy $\mathrm{NC}$ for the multicast relay network in Fig. 1 can be specialized from [31, Theorem 1] as follows

$$
\begin{aligned}
\sum_{k \in S} R(k)<\min _{d \in D}\{ & I\left(X(S) ; \hat{Y}\left(S^{c}\right) Y(d) \mid X\left(S^{c}\right) Q\right) \\
& \left.-I\left(Y(S) ; \hat{Y}(S) \mid X(T) \hat{Y}\left(S^{c}\right) Y(d) Q\right)\right\}
\end{aligned}
$$

where $\hat{Y}$ is the compressed versions of $Y, Q$ is the timesharing parameter, $S, S^{c}$ are any pair of complementary subsets of $T$, i.e., $S \cup S^{c}=T$ and $S \cap S^{c}=\emptyset$, with

$$
\begin{aligned}
& X\left(\mathcal{S}_{1}\right)=\left\{X_{1}, X_{s 1}\right\}, X\left(\mathcal{S}_{2}\right)=\left\{X_{2}, X_{s 2}\right\}, X(\mathcal{R})=X_{r}, \\
& X(T)=\left\{X_{1} X_{2} X_{r} X_{s 1} X_{s 2}\right\}, Y\left(\mathcal{S}_{1}\right)=Y_{s 1}, Y\left(\mathcal{S}_{2}\right)=Y_{s 2}, \\
& Y(\mathcal{R})=Y_{r}, Y\left(\mathcal{D}_{1}\right)=Y_{1}, Y\left(\mathcal{D}_{2}\right)=Y_{2},
\end{aligned}
$$

and the joint probability partitioned as

$$
\begin{aligned}
p(q) p\left(x_{1} \mid q\right) & p\left(x_{2} \mid q\right) p\left(x_{r} \mid q\right) p\left(x_{s 1} \mid q\right) p\left(x_{s 2} \mid q\right) \\
& \times p\left(\hat{y}_{r} \mid x_{r}, y_{r}, q\right) p\left(\hat{y}_{s 1} \mid x_{1}, y_{s 1}, q\right) p\left(\hat{y}_{s 2} \mid x_{2}, y_{s 2}, q\right) .
\end{aligned}
$$

By setting $Q=\emptyset$ and $\hat{Y}_{r}=Y_{r}+\hat{Z}_{r}, \hat{Y}_{s 1}=Y_{s 1}+\hat{Z}_{1}, \hat{Y}_{s 2}=Y_{s 2}+\hat{Z}_{2}$ with $\hat{Z}_{r} \sim \mathcal{N}\left(0, \sigma_{r}^{2}\right), \quad \hat{Z}_{1} \sim \mathcal{N}\left(0, \sigma_{1}^{2}\right), \quad \hat{Z}_{2} \sim \mathcal{N}\left(0, \sigma_{2}^{2}\right), \quad$ and applying (1), (2) and (6) into (8), we can get (7). 


\section{Relaying as Network Coding with Source COOPERATION}

In contrast to relaying compression of each other's messages as in Sec. IV, the source nodes can also cooperate by sharing parts of their messages through the conferencing links. By exploiting rate-splitting [36], [37], we first partition each source message into two parts $W_{1}=\left[W_{1 c}, W_{1 p}\right], W_{2}=\left[W_{2 c}, W_{2 p}\right]$, and then divide all the four messages evenly into $B$ blocks $W_{1 c, t}, W_{1 p, t}, W_{2 c, t}, W_{2 p, t}$, each with $n R_{1 c}, n R_{1 p}$, $n R_{2 c}, n R_{2 p}$, bits, respectively. The transmission is completed in $B+2$ blocks $^{2}$, each with $n$ channel uses. During block $t-1$, the sources exchange $\left(W_{1 c, t}, W_{2 c, t}\right)$ over the conferencing links at rate $R_{1 c} \leq C_{12}$ and $R_{2 c} \leq C_{21}$, respectively, to formulate a common message $W_{c, t}=\left[W_{1 c, t}, W_{2 c, t}\right]$; during block $t, \mathcal{S}_{1}$ broadcasts $\left[W_{c, t}, W_{1 p, t}\right]$ and $\mathcal{S}_{2}$ broadcasts $\left[W_{c, t}, W_{2 p, t}\right]$ over the channel in cooperation with the relay's transmission. Both DF relaying and AF relaying are considered here.

\section{A. Partial-Decode-and-Forward Relaying with Linear Net- work Coding $(p D F+L N C)$}

Unlike these cooperative strategies with DF relaying proposed in [32], $\mathcal{R}$ here only needs to decode and forward some or all of the messages $\left(W_{1 p, t}, W_{2 p, t}, W_{c, t}\right)$ depending on the channel quality, owning to the existence of cross-links. We propose a hybrid coding scheme termed partial-decodeand-forward based linear network coding $(\mathrm{pDF}+\mathrm{LNC})$. It essentially performs rate-splitting at the source nodes to exchange messages, partial decoding and LNC at the relay to reduce the rate constraints and superpose the decoded messages, and joint decoding at the destinations to enlarge the rate region. The codebook generation and encoding/decoding process are a natural extension of [21, Theorem 1]. Given i.i.d. random variables $V_{1 p}, V_{2 p}, V_{c} \sim \mathcal{N}(0,1)$, we first generate independent codebooks $\left\{V_{1 p}^{(n)}\right\},\left\{V_{2 p}^{(n)}\right\}$ and $\left\{V_{c}^{(n)}\right\}$, each of size $2^{n R_{1 p}}, 2^{n R_{2 p}}$ and $2^{n R_{c}}$, respectively. Then, for each index $k \in\left\{1, \ldots, 2^{n R_{1 p}}\right\}$, we generate independently $2^{n R_{1 p}}$ codewords $X_{1 p}^{(n)}$ using distribution $\prod p\left(x_{1 p} \mid v_{1 p}(k)\right)$, and label the codewords as $X_{1 p}^{(n)}(n, k)$, where $n \in\left\{1, \ldots, 2^{n R_{1 p}}\right\}$. We generate $X_{2 p}^{(n)}$ and $X_{c}^{(n)}$ in a similar way. At block $t, \mathcal{S}_{1}$ transmits $\left[W_{c, t}, W_{1 p, t}\right]$ and $\mathcal{S}_{2}$ transmits $\left[W_{c, t}, W_{2 p, t}\right]$ in cooperation with the relay's transmission as follows

$$
\begin{aligned}
X_{r, t}^{(n)}= & \sqrt{\alpha_{r}^{\prime}} V_{1 p}^{(n)}\left(W_{1 p, t-1}\right)+\sqrt{\alpha_{r}^{\prime \prime}} V_{2 p}^{(n)}\left(W_{2 p, t-1}\right) \\
& +\sqrt{1-\alpha_{r}^{\prime}-\alpha_{r}^{\prime \prime}} V_{c}^{(n)}\left(W_{c, t-1}\right), \\
X_{1, t}^{(n)}= & \sqrt{\alpha_{1}} X_{1 p}^{(n)}\left(W_{1 p, t}, W_{1 p, t-1}\right)+\sqrt{\alpha_{2}} X_{c}^{(n)}\left(W_{c, t}, W_{c, t-1}\right) \\
& +\sqrt{\alpha_{3}} V_{1 p}^{(n)}\left(W_{1 p, t-1}\right)+\sqrt{\alpha_{4}} V_{c}^{(n)}\left(W_{c, t-1}\right), \\
X_{2, t}^{(n)}= & \sqrt{\alpha_{5}} X_{2 p}^{(n)}\left(W_{2 p, t}, W_{2 p, t-1}\right)+\sqrt{\alpha_{6}} X_{c}^{(n)}\left(W_{c, t}, W_{c, t-1}\right) \\
& +\sqrt{\alpha_{7}} V_{2 p}^{(n)}\left(W_{2 p, t-1}\right)+\sqrt{\alpha_{8}} V_{c}^{(n)}\left(W_{c, t-1}\right),
\end{aligned}
$$

where $0 \leq \alpha_{r}^{\prime}, \alpha_{r}^{\prime \prime}, \alpha_{1}, \alpha_{2}, \alpha_{3}, \alpha_{4}, \alpha_{5}, \alpha_{6}, \alpha_{7}, \alpha_{8} \leq 1$ are power allocation parameters with $\alpha_{1}+\alpha_{2}+\alpha_{3}+\alpha_{4}=1$ and $\alpha_{5}+\alpha_{6}+\alpha_{7}+\alpha_{8}=1$. The encoding/decoding process when $\mathcal{R}$ decodes all messages with $B=3$ is illustrated in Table I. $\mathcal{R}$

\footnotetext{
${ }^{2}$ The first block $(t=0)$ involves only message exchanging via error-free bit-pipes but no transmission over the relay channel.
}

recovers $\left(W_{1 p, t}, W_{2 p, t}, W_{c, t}\right)$ jointly at the end of block $t$ by forward decoding [16] based on $Y_{r, t}^{(n)}$ after cancelling out $\left(W_{1 p, t-1}, W_{2 p, t-1}, W_{c, t-1}\right)$. The destinations carry out backward decoding [38]: the received signal $Y_{1, B+1}^{(n)}\left(Y_{2, B+1}^{(n)}\right)$ only depends on $\left(W_{1 p, B}, W_{2 p, B}, W_{c, B}\right)$, which can be retrieved by a joint typicality decoder; then we proceed to $Y_{1, B}^{(n)}\left(Y_{2, B}^{(n)}\right)$ and repeat this process backwards until all messages are recovered.

Proposition 6: Define $T=\{1 p, 2 p, c\}, T_{Q} \subseteq T$ and $T_{Q} \neq \emptyset$, the achievable rate region for $\mathrm{pDF}+\mathrm{LNC}$ is the union over all $\left(R_{1}, R_{2}\right)$ satisfying $R_{1} \geq 0, R_{2} \geq 0$, and

$$
\left\{\begin{array}{l}
0 \leq R_{1 c} \leq C_{12}, \quad 0 \leq R_{2 c} \leq C_{21}, R_{1 p} \geq 0, R_{2 p} \geq 0, \\
R_{1}=R_{1 p}+R_{1 c}, R_{2}=R_{2 p}+R_{2 c}, R_{c}=R_{1 c}+R_{2 c}, \\
R(S \subseteq T)<\min _{d \in\{1,2\}} I\left(X(S) X_{r} ; Y_{d} \mid X\left(S^{c}\right) V\left(S^{c}\right)\right), \\
R\left(S_{Q} \subseteq T_{Q}\right)<I\left(X\left(S_{Q}\right) ; Y_{r} \mid X\left(S_{Q}^{c}\right) V\left(T_{Q}\right)\right),
\end{array}\right.
$$

where $R(S)=\sum_{k \in S} R_{k}, S^{c}\left(S_{Q}^{c}\right)$ is the complementary subset of $S\left(S_{Q}\right)$ with $S \cup S^{c}=T\left(S_{Q} \cup S_{Q}^{c}=T_{Q}\right)$, the union is taken over all the power allocation parameters, and over all possible rate constraints $\left(R_{1 p}, R_{2 p}, R_{c}\right)$ that are determined by the corresponding partial-DF cooperation strategies indicated by $T_{Q}$. Intermediate variables $R_{1 p}, R_{2 p}, R_{c}, R_{1 c}$ and $R_{2 c}$ can be easily removed by performing Fourier-Motzkin elimination.

Proof: Proof outline. There are 7 different partial decoding options at the relay, namely, decoding only $W_{1 p, t}$, $W_{2 p, t}, W_{c, t},\left(W_{1 p, t}, W_{2 p, t}\right),\left(W_{1 p, t}, W_{c, t}\right),\left(W_{2 p, t}, W_{c, t}\right)$, or $\left(W_{1 p, t}, W_{2 p, t}, W_{c, t}\right)$, resulting in 7 different rate constraints $\left(R_{1 p}, R_{2 p}, R_{c}\right)$. We therefore introduce an auxiliary random variable $Q$ to indicate different partial DF strategies and any combinations of them by arbitrary time-sharing. If the relay decodes $\left(W_{1 p, t}, W_{2 p, t}, W_{c, t}\right)$ (i.e., $\left.T_{Q}=T\right)$, by performing forward decoding [16] at the relay and backward decoding [38] at destinations, we can get from [21, Theorem 1] that

$$
\begin{aligned}
& R(S \subseteq T)<I\left(X(S) ; Y_{r} \mid X\left(S^{c}\right) X_{r}\right), \\
& R(S \subseteq T)<\min _{d \in\{1,2\}} I\left(X(S) X_{r} ; Y_{d} \mid X\left(S^{c}\right) V\left(S^{c}\right)\right),
\end{aligned}
$$

with variables defined as in (9) and (1). By enforcing $p\left(x_{1 p} \mid v_{1 p}\right), p\left(x_{2 p} \mid v_{2 p}\right)$, and $p\left(x_{c} \mid v_{c}\right)$ to be normal distribution, and applying the fact that $V_{1 p}, V_{2 p}, V_{c} \sim \mathcal{N}(0,1)$ into (9), all the mutual information constraints in (11) can be translated into corresponding $\mathcal{C}(\cdot)$ expressions, which are omitted here due to space limitations.

If the relay only decodes $W_{c, t}\left(T_{Q}=\{c\}\right)$, we have

$$
\begin{gathered}
R(S \subseteq T)<\min _{d \in\{1,2\}} I\left(X(S) X_{r} ; Y_{d} \mid X\left(S^{c}\right) V\left(S^{c}\right)\right), \\
R_{c}<I\left(X_{c} ; Y_{r} \mid V_{c}\right)
\end{gathered}
$$

with variables defined as in (9) and (1) but with $\alpha_{r}^{\prime}=\alpha_{r}^{\prime \prime}=$ $\alpha_{3}=\alpha_{7}=0$. The case when the relay only decodes $W_{1 p, t}$ (for $T_{Q}=\{1 p\}$ ) or $W_{2 p, t}$ (for $T_{Q}=\{2 p\}$ ) is handled similarly.

If the relay decodes $W_{1 p, t}, X_{2 p, t}$ but not $W_{c, t}$ (for $T_{Q}=$ $\{1 p, 2 p\})$, we can obtain

$$
\begin{aligned}
R(S \subseteq T) & <\min _{d \in\{1,2\}} I\left(X(S) X_{r} ; Y_{d} \mid X\left(S^{c}\right) V\left(S^{c}\right)\right), \\
R_{1 p} & <I\left(X_{1 p} ; Y_{r} \mid X_{2 p} V_{1 p} V_{2 p}\right), \\
R_{2 p} & <I\left(X_{2 p} ; Y_{r} \mid X_{1 p} V_{1 p} V_{2 p}\right), \\
R_{1 p}+R_{2 p} & <I\left(X_{1 p} X_{2 p} ; Y_{r} \mid V_{1 p} V_{2 p}\right),
\end{aligned}
$$


TABLE I

ILLUSTRATION OF THE ENCODING/DECODING PROCESS FOR PDF + LNC WITH $W_{c, t}=\left[W_{1 c, t}, W_{2 c, t}\right]$ FOR $B=3$ AND FULL DECODING AT THE RELAY.

\begin{tabular}{|c|c|c|c|c|c|}
\hline$t=$ & 0 & 1 & 2 & 3 & 4 \\
\hline$\rightleftharpoons$ & $W_{1 c, 1} \Leftrightarrow W_{2 c, 1}$ & $W_{1 c, 2} \Leftrightarrow W_{2 c, 2}$ & $W_{1 c, 3} \Leftrightarrow W_{2 c, 3}$ & $/$ & $/$ \\
\hline $\mathcal{S}_{1}$ transmits & $/$ & $W_{1 p, 1}, W_{c, 1}$ & $\left(W_{1 p, 1}, W_{1 p, 2}, W_{c, 1}, W_{c, 2}\right)$ & $\left(W_{1 p, 2}, W_{1 p, 3}, W_{c, 2}, W_{c, 3}\right)$ & $W_{1 p, 3}, W_{c, 3}$ \\
\hline $\mathcal{S}_{2}$ transmits & $/$ & $W_{2 p, 1}, W_{c, 1}$ & $\left(W_{2 p, 1}, W_{2 p, 2}, W_{c, 1}, W_{c, 2}\right)$ & $\left(W_{2 p, 2}, W_{2 p, 3}, W_{c, 2}, W_{c, 3}\right)$ & $W_{2 p, 3}, W_{c, 3}$ \\
\hline $\mathcal{R}$ decodes & $/$ & $W_{1,1}, W_{2,1} \rightarrow$ & $W_{1,2}, W_{2,2} \rightarrow$ & $W_{1,3}, W_{2,3}$ & $/$ \\
\hline $\mathcal{R}$ transmits & $/$ & $/$ & $W_{1 p, 1}, W_{2 p, 1}, W_{c, 1}$ & $W_{1 p, 2}, W_{2 p, 2}, W_{c, 2}$ & $W_{1 p, 3}, W_{2 p, 3}, W_{c, 3}$ \\
\hline $\mathcal{D}_{1} / \mathcal{D}_{2}$ decodes & $/$ & $/$ & $W_{1,1}, W_{2,1}$ & $\leftarrow W_{1,2}, W_{2,2}$ & $\leftarrow W_{1,3}, W_{2,3}$ \\
\hline
\end{tabular}

TABLE II

ILLUSTRATION OF THE ENCODING/DECODING PROCESS FOR AF+ANC WITH $W_{c, t}=\left[W_{1 c, t}, W_{2 c, t}\right]$ FOR $B=3$.

\begin{tabular}{|c|c|c|c|c|c|}
\hline$t=$ & 0 & 1 & 2 & 3 & 4 \\
\hline$\rightleftharpoons$ & $W_{1 c, 1} \Leftrightarrow W_{2 c, 1}$ & $W_{1 c, 2} \Leftrightarrow W_{2 c, 2}$ & $W_{1 c, 3} \Leftrightarrow W_{2 c, 3}$ & $/$ & $/$ \\
\hline $\mathcal{S}_{1}$ transmits & $/$ & $\left(W_{1 p, 1}, W_{c, 1}\right)$ & $\left(W_{1 p, 2}, W_{c, 2}\right)$ & $\left(W_{1 p, 3}, W_{c, 3}\right)$ & \\
\hline $\mathcal{S}_{2}$ transmits & $/$ & $\left(W_{2 p, 1}, W_{c, 1}\right)$ & $\left(W_{2 p, 2}, W_{c, 2}\right)$ & $\left(W_{2 p, 3}, W_{c, 3}\right)$ & \\
\hline $\mathcal{R}$ relays & $/$ & $/$ & $W_{1 p, 1}, W_{2 p, 1}, W_{c, 1}$ & $W_{1 p, 2}, W_{2 p, 2}, W_{c, 2}$ & $W_{1 p, 3}, W_{2 p, 3}, W_{c, 3}$ \\
\hline $\mathcal{D}_{1} / \mathcal{D}_{2}$ decodes & $/$ & $/$ & $W_{1,1}, W_{2,1} \rightarrow$ & $W_{1,2}, W_{2,2} \rightarrow$ & $W_{1,3}, W_{2,3}$ \\
\hline
\end{tabular}

with variables defined as in (9) and (1) but with $\alpha_{r}^{\prime}+\alpha_{r}^{\prime \prime}=1$ and $\alpha_{4}=\alpha_{8}=0$. It is similar for scenarios when the relay does not decode $W_{1 p, t}$ (for $\left.T_{Q}=\{2 p, c\}\right)$ or $W_{2 p, t}$ (for $T_{Q}=\{1 p, c\}$ ).

For other values of $Q$, different partial DF strategies are used in a time-sharing fashion. The achievable rate region in (10) is therefore the union of all the different regions resulting from different partial decoding strategies.

Remark 2: The $\mathrm{pDF}+\mathrm{LNC}$ strategy requires a smart relay which can adopt a proper encoding/decoding scheme depending on the effective link SNR $\gamma$, in addition to a powerful joint typicality decoder. Based on the design metric (e.g. maximizing the sum-rate) and the values of $\gamma_{i, j}$, the same optimization process can be carried out at both the relay and source nodes, resulting in an operation point $\left(R_{1}, R_{2}\right)$ on the boundary of the achievable rate region together with a group of operating parameters $\left(Q, R_{1 p}, R_{2 p}, R_{1 c}, R_{2 c}, \alpha_{r}^{\prime}, \alpha_{r}^{\prime \prime}, \alpha_{1}, \alpha_{2}, \alpha_{3}, \alpha_{4}, \alpha_{5}, \alpha_{6}, \alpha_{7}, \alpha_{8}\right)$. Ideally such optimization process can be carried out on the fly to adaptively update the operating parameters. For practical implementation however, we need to form a lookup table for $\mathcal{R}$ which contains $\left(Q, R_{1 p}, R_{2 p}, R_{1 c}, R_{2 c}, \alpha_{r}^{\prime}, \alpha_{r}^{\prime \prime}\right)$ and is indexed by quantized link SNR $\tilde{\gamma}_{i j}, i, j=1,2, r$. The lookup tables for $\mathcal{S}_{1}$ and $\mathcal{S}_{2}$ are created in a similar way. Note that the quantization should satisfy $\tilde{\gamma} \leq \gamma$ to avoid link outage and hence results in a loss of spectrum efficiency. If the quantization resolution is properly selected, the complexity of implementing a lookup table can be marginal compared to the joint typicality decoder equipped by the DF relay.

\section{B. Amplify-and-Forward as Analog Network Coding $(A F+A N C)$}

The proposed $\mathrm{pDF}+\mathrm{LNC}$ strategy requires a powerful relay node. If the functionality of the relay cannot support encoding/decoding or interference cancellation, neither the extension of Noisy NC in Sec. IV nor pDF $+\mathrm{LNC}$ can be used. As suggested by [29], [30], AF relaying as analog NC (AF+ANC) is an attractive option in the high SNR regime. In this setup, the relay forwards a scaled version of the signal received during the previous period. Three independent random codebooks $\left\{V_{1 p, t}^{(n)}\right\}$ of size $2^{n R_{1 p}},\left\{V_{2 p, t}^{(n)}\right\}$ of size $2^{n R_{2 p}}$, and $\left\{V_{c, t}^{(n)}\right\}$ of size $2^{n\left(R_{1 c}+R_{2 c}\right)}$, are generated to encode $W_{1 p, t}, W_{2 p, t}$ and $W_{c, t}$, respectively. At block $t$, the transmitted signals are

$$
\begin{aligned}
& X_{1, t}^{(n)}= \sqrt{\bar{\alpha}_{1}} V_{1 p, t}^{(n)}\left(W_{1 p, t}\right)+\sqrt{\alpha_{1}} V_{c, t}^{(n)}\left(W_{c, t}\right), \\
& X_{2, t}^{(n)}=\sqrt{\bar{\alpha}_{2}} V_{2 p, t}^{(n)}\left(W_{2 p, t}\right)+\sqrt{\alpha_{2}} V_{c, t}^{(n)}\left(W_{c, t}\right), \\
& X_{r, t}^{(n)}=\beta\left(\sqrt{\gamma_{1 r} \bar{\alpha}_{1}} V_{1 p, t-1}^{(n)}+\sqrt{\gamma_{2 r} \bar{\alpha}_{2}} V_{2 p, t-1}^{(n)}\right. \\
&\left.+\left(\sqrt{\gamma_{1 r} \alpha_{1}}+\sqrt{\gamma_{2 r} \alpha_{2}}\right) V_{c, t-1}^{(n)}+Z_{r, t-1}^{(n)}\right),
\end{aligned}
$$

where $0 \leq \alpha_{1}, \alpha_{2} \leq 1$ are power allocation parameters with $\bar{\alpha}_{1}=1-\alpha_{1}$ and $\bar{\alpha}_{2}=1-\alpha_{2}$. $\beta$ is the amplifying factor at the relay to satisfy the power constraint (2), i.e.,

$$
\beta^{2}=\frac{1}{E\left[\operatorname{Var}\left(Y_{r, t}\right)\right]}=\frac{1}{1+\gamma_{1 r}+\gamma_{2 r}+2 \sqrt{\gamma_{1 r} \gamma_{2 r} \alpha_{1} \alpha_{2}}} .
$$

Note that $\beta$ is defined in a different way in [30] to guarantee the SNR level at the destination nodes after multiple-hop AF relaying. The destination nodes $\mathcal{D}_{1}$ and $\mathcal{D}_{2}$ perform sliding window [39] joint decoding: at the end of block $t+1$, assuming $W_{1, t-1}$ and $W_{2, t-1}$ have been decoded successfully, $\mathcal{D}_{1}$ can jointly decode $W_{1, t}$ and $W_{2, t}$ from $\left(Y_{1, t}^{(n)}, Y_{1, t+1}^{(n)}\right)$ and $\mathcal{D}_{2}$ can decode based on $\left(Y_{2, t}^{(n)}, Y_{2, t+1}^{(n)}\right)$. The encoding/decoding process is illustrated in Table II.

Proposition 7: Define $T=\{1 p, 2 p, c\}$ and a pair of its complementary subsets $S$ and $S^{c}$, i.e. $S \cup S^{c}=T$ and $S \cap S^{c}=\emptyset$, the achievable rate region for $\mathrm{AF}+\mathrm{ANC}$ is the union over all $\left(R_{1}, R_{2}\right)$ satisfying $R_{1} \geq 0, R_{2} \geq 0$, and

$\left\{\begin{array}{l}0 \leq R_{1 c} \leq C_{12}, \quad 0 \leq R_{2 c} \leq C_{21}, R_{1 p} \geq 0, R_{2 p} \geq 0, \\ R_{1}=R_{1 p}+R_{1 c}, R_{2}=R_{2 p}+R_{2 c}, R_{c}=R_{1 c}+R_{2 c}, \\ \sum_{k \in S} R_{k}<\min _{d \in\{1,2\}} I\left(V_{t}(S) ; Y_{d, t} Y_{d, t+1} \mid V_{t-1}(T) V_{t}\left(S^{c}\right)\right),\end{array}\right.$

where $V_{t-1}(T)=\left\{V_{1 p, t-1}, V_{2 p, t-1}, V_{c, t-1}\right\}$, and the union is taken over all subsets $S \subseteq T$ and over all power allocation parameters $0 \leq \alpha_{1}, \alpha_{2} \leq 1$. The compact rate region described by $\left(R_{1}, R_{2}\right)$ can be straightforwardly obtained by performing Fourier-Motzkin elimination to remove the intermediate variables $R_{1 p}, R_{2 p}, R_{c}, R_{1 c}$ and $R_{2 c}$.

Proof: Proof outline.

Since $X_{1, t}-V_{c, t}-X_{2, t}$ form a Markov chain, by sliding 

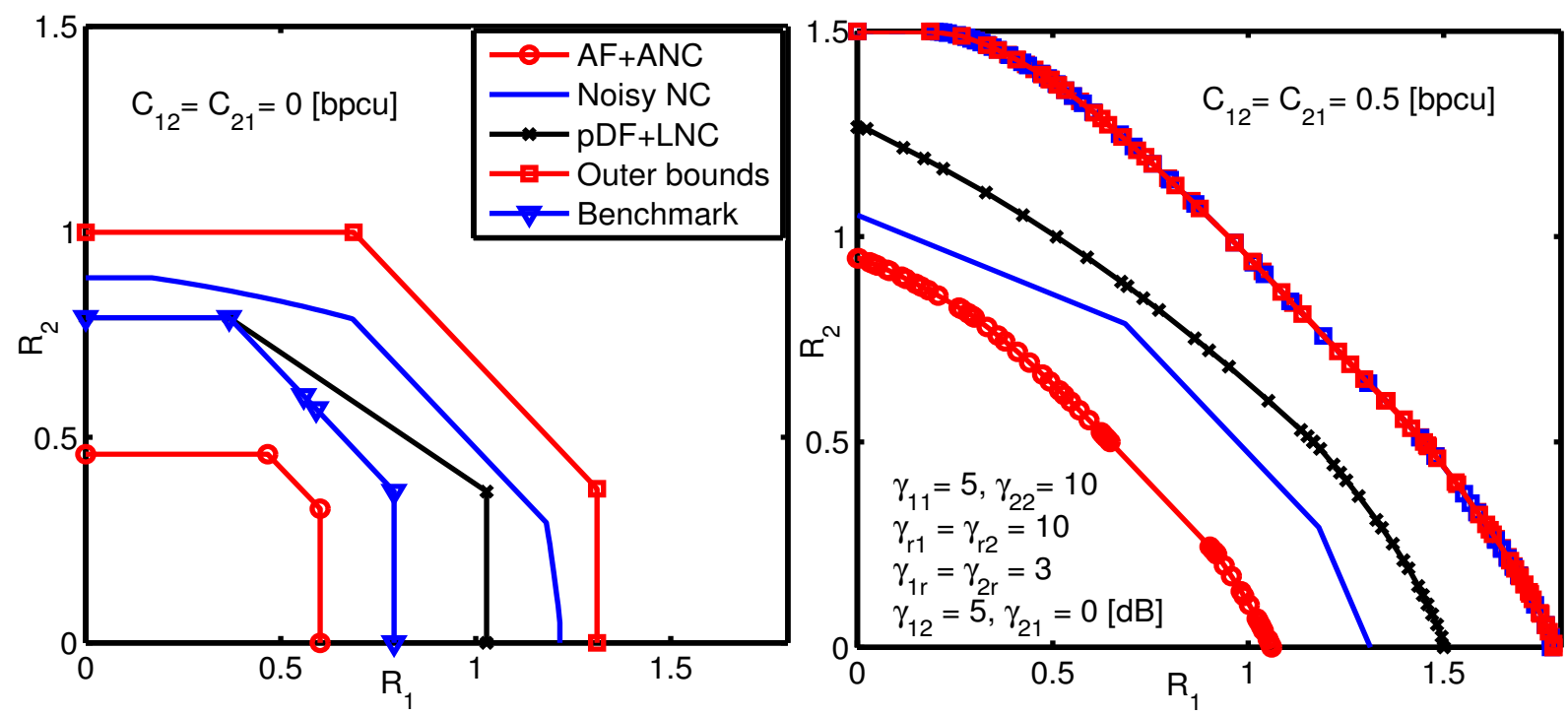

Fig. 3. Achievable rate region of $\mathrm{AF}+\mathrm{ANC}$, Noisy $\mathrm{NC}$, and $\mathrm{pDF}+\mathrm{LNC}$, as well as the capacity outer bounds, with (right) and without (left) conferencing links. The benchmark is obtained from [24, Proposition 4] for a DF relay without source cooperation.

window joint decoding based on $\left(Y_{1, t}^{(n)}, Y_{1, t+1}^{(n)}\right)$ at $\mathcal{D}_{1}$ and $\left(Y_{2, t}^{(n)}, Y_{2, t+1}^{(n)}\right)$ at $\mathcal{D}_{2}$, respectively, we will get

$$
\sum_{k \in S} R_{k}<\min _{d \in\{1,2\}} I\left(V_{t}(S) ; Y_{d, t} Y_{d, t+1} \mid V_{t-1}(T) V_{t}\left(S^{c}\right)\right) .
$$

Now we will show that all the mutual information terms in (16) are simultaneously maximized by Gaussian distributed signals $V_{1 p, t}, V_{2 p, t}, V_{c, t}$. Note that for $S=T$ and $d=1$ we have

$$
\begin{aligned}
& I\left(V_{1 p, t} V_{2 p, t} V_{c, t} ; Y_{1, t} Y_{1, t+1} \mid V_{1 p, t-1} V_{2 p, t-1} V_{c, t-1}\right) \\
& =h\left(Y_{1, t}, Y_{1, t+1} \mid V_{1 p, t-1} V_{2 p, t-1} V_{c, t-1}\right)-h\left(\tilde{Z}_{t}\right) \\
& \quad-h\left(\sqrt{\gamma_{11}} X_{1, t+1}+\sqrt{\gamma_{21}} X_{2, t+1}+\tilde{Z}_{t+1}\right) \\
& \leq \frac{1}{2} \log \left(\left|\boldsymbol{K}_{y}\right|\right)-\frac{1}{2} \log \left(\sigma_{1}^{2}\right) \\
& \quad-\frac{1}{2} \log \left(\sigma_{1}^{2}+\operatorname{Var}\left(\sqrt{\gamma_{11}} X_{1, t+1}+\sqrt{\gamma_{21}} X_{2, t+1}\right)\right) \\
& =\mathcal{C}\left(\frac{\gamma_{11}+\gamma_{21}+2 \sqrt{\gamma_{11} \gamma_{21} \alpha_{1} \alpha_{2}}}{1+\beta^{2} \gamma_{r 1}}\right. \\
& \quad+\frac{\beta^{2} \gamma_{r 1}\left(\gamma_{1 r}+\gamma_{2 r}+2 \sqrt{\gamma_{1 r} \gamma_{2 r} \alpha_{1} \alpha_{2}}\right)}{1+\beta^{2} \gamma_{r 1}+\gamma_{11}+\gamma_{21}+2 \sqrt{\gamma_{11} \gamma_{21} \alpha_{1} \alpha_{2}}} \\
& \left.+\frac{\beta^{2} \gamma_{r 1}\left(1-\alpha_{1} \alpha_{2}\right)\left(\sqrt{\gamma_{11} \gamma_{2 r}}-\sqrt{\gamma_{21} \gamma_{1 r}}\right)^{2}}{\left(1+\beta^{2} \gamma_{r 1}\right)\left(1+\beta^{2} \gamma_{r 1}+\gamma_{11}+\gamma_{21}+2 \sqrt{\gamma_{11} \gamma_{21} \alpha_{1} \alpha_{2}}\right)}\right)
\end{aligned}
$$

where $\tilde{Z}_{1, t}, t=1, \ldots, B+1$ are i.i.d. Gaussian with zero-mean and variance $\sigma_{1}^{2}=1+\beta^{2} \gamma_{r 1}$, and $\boldsymbol{K}_{y}$ is the conditional covariance matrix of $\left(Y_{1, t}, Y_{1, t+1}\right)$ given $\left(V_{1 p, t-1} V_{2 p, t-1} V_{c, t-1}\right)$. The inequality in (17) comes from the Maximum Entropy Lemma and the Entropy Power Inequality [35], with equality achieved by the joint Gaussian distribution, and the equality in (18) is obtained by applying (14) into (1).

Following a similar procedure, we can show that all the mutual information items in (16) are simultaneously maximized by Gaussian distributions. Then it is straightforward to translate them to corresponding $\mathcal{C}(\cdot)$ expressions, which are omitted here owing to space limitations.

\section{Numerical Results}

We illustrate the new inner and outer bounds to the capacity region based on numerical computation, with channel SNR chosen heuristically. As stated in Sec. V-A, computation of the rate region of $\mathrm{pDF}+\mathrm{LNC}$ requires a union operation over eight independent auxiliary variables and seven partial decoding combinations, making it hard to characterize the exact inner bound numerically. Unless stated otherwise, in the following results we simply set $\alpha_{3}=\alpha_{4}=0$ and $\alpha_{7}=\alpha_{8}=0$ in (9), i.e. no source-relay cooperation, to lower bound the performance of pDF+LNC.

The benefit of using the conferencing links has been illustrated in Fig. 3. Without source cooperation, achievable rates for $\mathrm{AF} / \mathrm{DF}$ relaying based schemes are limited by noise propagation and decoding constraints when the source-relay link is poor. When source cooperation is possible, these constraints can be greatly reduced. The gap between outer and inner bounds is within 0.2 bits for no cooperation and within 0.3 bits for $C_{12}=C_{21}=0.5$ bits per channel use (bpcu). The difference between different outer bounds is within 0.01 bits in both cases. A benchmark scheme based on DF relaying with no source conferencing from [24, Proposition 4] with $R_{3}=0$ has been plotted in Fig. 3 (left) for reference. The gain of $\mathrm{pDF}+\mathrm{LNC}$ (with source-relay cooperation) over the benchmark is due to partial decoding at the relay.

In Fig. 4 we compare the rate regions for weak/strong relaydestination links with asymmetric conferencing rates. With message exchange in $\mathrm{AF}+\mathrm{ANC}$, the transmitted signals by $\mathcal{S}_{1}$ and $\mathcal{S}_{2}$ contain the same codewords $U_{t}^{(n)}\left(W_{c, t}\right)$, as shown in (14), which can coherently add up at the relay and destinations. The simple AF based scheme can therefore outperform noisy $\mathrm{NC}$ in some regions, where the coherent combining gain is dominant. The gap between inner and outer bounds is within 0.3 bits when the relay-destination links are weak, but decreases to within 0.1 bits for strong relay-destination links. The difference between different outer bounds is negligible. 


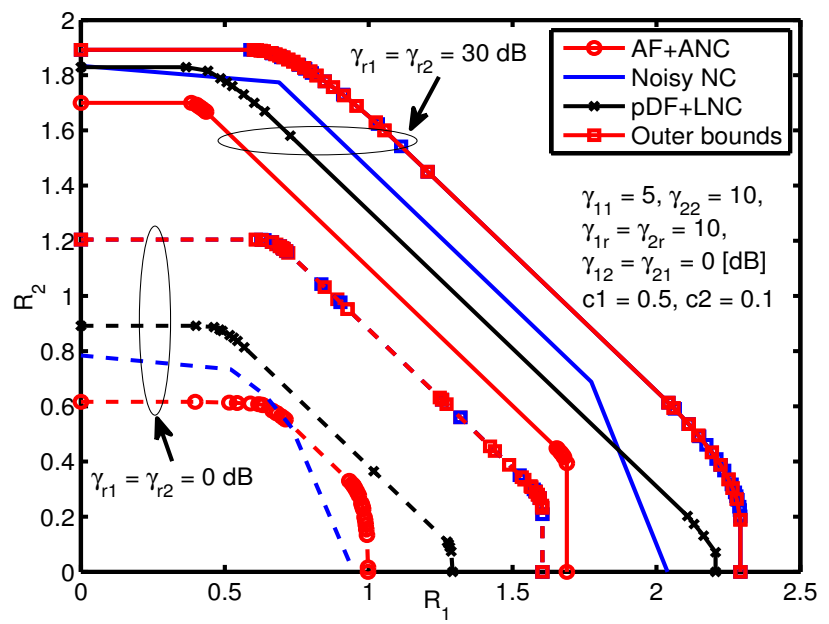

Fig. 4. Achievable rate region of AF+ANC, Noisy NC, and pDF+LNC, as well as the capacity outer bounds, for channels setups with direct links $\gamma_{11}=$ $5 \mathrm{~dB}$ and $\gamma_{22}=10 \mathrm{~dB}$, cross-links $\gamma_{12}=\gamma_{21}=0 \mathrm{~dB}$, source-relay links $\gamma_{1 r}=\gamma_{2 r}=10 \mathrm{~dB}$ and weak/strong relay-destination links, with asymmetric conferencing rates $C_{12}=0.5, C_{21}=0.1$ bits per channel use.

\section{CONCLUSION}

We have studied the capacity region for a wireless multicast relay network with partially cooperating source nodes. We have provided two genie-aided outer bounds by introducing two new lemmas on conditional (co-)variance. We also have provided three cooperative relaying schemes, namely, noisy NC with source cooperation, partial DF relaying with LNC, and ANC. We have characterized the achievable rate regions and demonstrated that these can be greatly enlarged with the help of the conferencing links, especially for $\mathrm{AF}+\mathrm{ANC}$ and $\mathrm{pDF}+\mathrm{LNC}$. The gap between inner and outer bounds is small, within 0.3 bits in the scenarios we have considered. By adaptively exploiting these cooperation schemes based on channel quality information, we may achieve a better inner bound and therefore a smaller gap. We have also pointed out the limitation of noisy $\mathrm{NC}$ on maximizing the sum-rate and shown that $\mathrm{AF}+\mathrm{ANC}$ can even outperform noisy $\mathrm{NC}$ when the coherent combining gain is dominant.

\section{APPENDiX A}

\section{ProOF OF PROPOSITION 2}

We first present two lemmas that will be used in our proof.

Lemma 1: For random variables $X, Y, Z$ on the same probability space, each with finite variance, and $Y, Z$ are independent, we have

$$
\begin{aligned}
& E[\operatorname{Var}(X \mid Y)]=E[\operatorname{Var}(X \mid Y, Z)]+E[\operatorname{Var}(E[X \mid Z] \mid Y)] . \\
& \text { Proof: } \\
& \quad E[\operatorname{Var}(X \mid Y)]-E[\operatorname{Var}(X \mid Y, Z)] \\
& \quad=E\left(E^{2}[X \mid Y, Z]\right)-E\left(E^{2}[X \mid Y]\right) \\
& \quad \stackrel{(a)}{=} E\left(T^{2}\right)-E\left(E^{2}[T \mid Y]\right)=E[\operatorname{Var}(T \mid Y)] \\
& \quad=E[\operatorname{Var}(E[X \mid Y, Z] \mid Y)] \stackrel{(b)}{=} E[\operatorname{Var}(E[X \mid Z] \mid Y)],
\end{aligned}
$$

where $(a)$ comes from variable substitution $T=E[X \mid Y, Z]$ and the fact that $E[T \mid Y]=E(E[X \mid Y, Z] \mid Y)=E[X \mid Y]$, and $(b)$ is due to $\operatorname{Var}(E[X \mid Y=y, Z] \mid Y=y)=\operatorname{Var}(E[X \mid Z] \mid Y=y)$.
Lemma 2: For random variables $X, Y, Z, U$ on the same probability space, each with finite variance, $Z, U$ are independent and $X-(Z, U)-Y$ is a Markov chain, then

$E[\operatorname{Cov}(X, Y \mid Z)]=E[\operatorname{Cov}(E[X \mid U], E[Y \mid U] \mid Z)]$.

Proof:

$$
\begin{aligned}
& E[\operatorname{Cov}(X, Y \mid Z)]=E(X Y)-E(E[X \mid Z] E[Y \mid Z]) \\
& =E(E[X Y \mid Z, U])-E(E(E[X \mid Z, U] \mid Z) E(E[Y \mid Z, U] \mid Z)) \\
& \stackrel{(c)}{=} E[\operatorname{Cov}(E[X \mid Z, U], E[Y \mid Z, U] \mid Z)] \\
& \stackrel{(d)}{=} E[\operatorname{Cov}(E[X \mid U], E[Y \mid U] \mid Z)],
\end{aligned}
$$

where $(c)$ is due to Markovicity and $(d)$ comes from $\operatorname{Cov}(E[X \mid z, U], E[Y \mid z, U] \mid z)=\operatorname{Cov}(E[X \mid U], E[Y \mid U] \mid z)$.

Now we are ready to prove Proposition 2. Note that

$$
\begin{aligned}
\frac{1}{n} \sum_{i=1}^{n} E\left[E^{2}\left(X_{1, i} \mid X_{r, i}\right)\right] & \leq \frac{1}{n} \sum_{i=1}^{n} E\left[E\left(X_{1, i}^{2} \mid X_{r, i}\right)\right] \\
& =\frac{1}{n} \sum_{i=1}^{n} E\left[X_{1, i}^{2}\right] \leq 1,
\end{aligned}
$$

we introduce an auxiliary variable $\alpha_{1} \in[0,1]$ as in [16], [32], [33] such that

$$
\bar{\alpha}_{1}=1-\alpha_{1}=\frac{1}{n} \sum_{i=1}^{n} E\left[E^{2}\left(X_{1, i} \mid X_{r, i}\right)\right] .
$$

It is easy to show that

$$
\begin{aligned}
& \frac{1}{n} \sum_{i=1}^{n} E\left[\operatorname{Var}\left(X_{1, i} \mid X_{r, i}\right)\right] \\
& =\frac{1}{n} \sum_{i=1}^{n} E\left[X_{1, i}^{2}\right]-\frac{1}{n} \sum_{i=1}^{n} E\left[E^{2}\left(X_{1, i} \mid X_{r, i}\right)\right] \leq \alpha_{1},
\end{aligned}
$$

where the inequality comes from the power constraint (2).

With the help of the genie, we have $X_{1}-\left(X_{r}, X_{s}\right)-X_{2}$ with $X_{r}$ independent of $X_{s}$. By Lemma 1 and the fact that $E\left[\operatorname{Var}\left(X_{1} \mid X_{r} X_{s}\right)\right] \leq E\left[\operatorname{Var}\left(X_{1} \mid X_{r}\right)\right]$, we define $\rho_{1} \in[0,1]$ with $\bar{\rho}_{1}=1-\rho_{1}$ such that

$$
\begin{aligned}
& \frac{1}{n} \sum_{i=1}^{n} E\left[\operatorname{Var}\left(X_{1, i} \mid X_{r, i} X_{s, i}\right)\right]=\bar{\rho}_{1} \alpha_{1}, \\
& \frac{1}{n} \sum_{i=1}^{n} E\left[\operatorname{Var}\left(E\left[X_{1, i} \mid X_{s, i}\right] \mid X_{r, i}\right)\right] \leq \rho_{1} \alpha_{1} .
\end{aligned}
$$

Similarly, we can define $\alpha_{2}, \rho_{2} \in[0,1]$ with $\bar{\alpha}_{2}=1-\alpha_{2}$, $\bar{\rho}_{2}=1-\rho_{2}$ such that

$$
\begin{aligned}
& \frac{1}{n} \sum_{i=1}^{n} E\left[E^{2}\left(X_{2, i} \mid X_{r, i}\right)\right]=\bar{\alpha}_{2}, \\
& \frac{1}{n} \sum_{i=1}^{n} E\left[\operatorname{Var}\left(X_{2, i} \mid X_{r, i}\right)\right] \leq \alpha_{2},
\end{aligned}
$$

and

$$
\begin{aligned}
& \frac{1}{n} \sum_{i=1}^{n} E\left[\operatorname{Var}\left(X_{2, i} \mid X_{r, i} X_{s, i}\right)\right]=\bar{\rho}_{2} \alpha_{2}, \\
& \frac{1}{n} \sum_{i=1}^{n} E \operatorname{Var}\left(E\left[X_{2, i} \mid X_{s, i}\right] \mid X_{r, i}\right) \leq \rho_{2} \alpha_{2} .
\end{aligned}
$$


Since $\operatorname{Cov}(X, Y) \leq \sqrt{\operatorname{Var}(X) \operatorname{Var}(Y)}$, we have

$$
\begin{aligned}
& \frac{1}{n} \sum_{i=1}^{n} \operatorname{Cov}\left[E\left(X_{1, i} \mid X_{r, i}\right), E\left(X_{2, i} \mid X_{r, i}\right)\right] \\
& \leq \frac{1}{n} \sum_{i=1}^{n} \sqrt{\operatorname{Var}\left[E\left(X_{1, i} \mid X_{r, i}\right)\right] \operatorname{Var}\left[E\left(X_{2, i} \mid X_{r, i}\right)\right]} \\
& \leq \sqrt{\frac{1}{n} \sum_{i=1}^{n} \operatorname{Var}\left[E\left(X_{1, i} \mid X_{r, i}\right)\right] \frac{1}{n} \sum_{i=1}^{n} \operatorname{Var}\left[E\left(X_{2, i} \mid X_{r, i}\right)\right]} \\
& \leq \sqrt{\bar{\alpha}_{1} \bar{\alpha}_{2}},
\end{aligned}
$$

where the second inequality is by the Cauchy-Schwarz inequality, and the last inequality is due to $\operatorname{Var}(X) \leq E\left(X^{2}\right)$ with substitution of (19) and (22). Applying the same procedure, we can further obtain

$\frac{1}{n} \sum_{i=1}^{n} \operatorname{Cov}\left(X_{1, i}, X_{r, i}\right)=\frac{1}{n} \sum_{i=1}^{n} \operatorname{Cov}\left(E\left[X_{1, i} \mid X_{r, i}\right], X_{r, i}\right) \leq \sqrt{\bar{\alpha}_{1}}$,

$\frac{1}{n} \sum_{i=1}^{n} \operatorname{Cov}\left(X_{2, i}, X_{r, i}\right)=\frac{1}{n} \sum_{i=1}^{n} \operatorname{Cov}\left(E\left[X_{2, i} \mid X_{r, i}\right], X_{r, i}\right) \leq \sqrt{\bar{\alpha}_{2}}$,

$$
\begin{aligned}
& \frac{1}{n} \sum_{i=1}^{n} E\left[\operatorname{Cov}\left(X_{1, i}, X_{2, i} \mid X_{r, i}\right)\right] \\
& =\frac{1}{n} \sum_{i=1}^{n} E\left[\operatorname{Cov}\left(E\left(X_{1, i} \mid X_{s, i}\right), E\left(X_{2, i} \mid X_{s, i}\right) \mid X_{r, i}\right)\right]
\end{aligned}
$$

$\leq \frac{1}{n} \sum_{i=1}^{n} \sqrt{E\left[\operatorname{Var}\left(E\left[X_{1, i} \mid X_{s, i}\right] \mid X_{r, i}\right)\right] E\left[\operatorname{Var}\left(E\left[X_{2, i} \mid X_{s, i}\right] \mid X_{r, i}\right)\right]}$

$$
\leq \sqrt{\rho_{1} \rho_{2} \alpha_{1} \alpha_{2}}
$$

where the equality in (27) is due to Lemma 2. By the Law of total covariance [40], we have

$$
\operatorname{Cov}(X, Y)=E[\operatorname{Cov}(X, Y \mid Z)]+\operatorname{Cov}(E[X \mid Z], E[Y \mid Z]),
$$

which, combined with (27) and (24), leads to

$$
\frac{1}{n} \sum_{i=1}^{n} \operatorname{Cov}\left(X_{1, i}, X_{2, i}\right) \leq \sqrt{\rho_{1} \rho_{2} \alpha_{1} \alpha_{2}}+\sqrt{\bar{\alpha}_{1} \bar{\alpha}_{2}}
$$

Similar to (27), we can obtain by Lemma 2 that

$$
\begin{aligned}
& \frac{1}{n} \sum_{i=1}^{n} E\left[\operatorname{Cov}\left(X_{1, i}, X_{2, i} \mid X_{s, i}\right)\right] \\
& =\frac{1}{n} \sum_{i=1}^{n} E\left[\operatorname{Cov}\left(E\left(X_{1, i} \mid X_{r, i}\right), E\left(X_{2, i} \mid X_{r, i}\right) \mid X_{s, i}\right)\right] \\
& \leq \frac{1}{n} \sum_{i=1}^{n} \sqrt{E\left[\operatorname{Var}\left(E\left(X_{1, i} \mid X_{r, i}\right) \mid X_{s, i}\right)\right] E\left[\operatorname{Var}\left(E\left(X_{2, i} \mid X_{r, i}\right) \mid X_{s, i}\right)\right]} \\
& \leq \frac{1}{n} \sum_{i=1}^{n} \sqrt{E\left(E^{2}\left(X_{1, i} \mid X_{r, i}\right)\right) E\left(E^{2}\left(X_{2, i} \mid X_{r, i}\right)\right)} \leq \sqrt{\bar{\alpha}_{1} \bar{\alpha}_{2}}
\end{aligned}
$$

where the second inequality comes from

$$
E[\operatorname{Var}(X \mid Z)]=E\left(X^{2}\right)-E\left(E^{2}(X \mid Z)\right) \leq E\left(X^{2}\right) .
$$

By Lemma 1 and (30), we can obtain

$$
\begin{aligned}
& \frac{1}{n} \sum_{i=1}^{n} E\left[\operatorname{Var}\left(X_{1, i} \mid X_{s, i}\right)\right] \\
& =\frac{1}{n} \sum_{i=1}^{n} E\left[\operatorname{Var}\left(X_{1, i} \mid X_{r, i}, X_{s, i}\right)\right]+E\left[\operatorname{Var}\left(E\left[X_{1, i} \mid X_{r, i}\right] \mid X_{s, i}\right)\right] \\
& \leq \frac{1}{n} \sum_{i=1}^{n} E\left[\operatorname{Var}\left(X_{1, i} \mid X_{r, i}, X_{s, i}\right)\right]+\frac{1}{n} \sum_{i=1}^{n} E\left(E^{2}\left[X_{1, i} \mid X_{r, i}\right]\right) \\
& \leq \bar{\rho}_{1} \alpha_{1}+\bar{\alpha}_{1}
\end{aligned}
$$

and

$$
\frac{1}{n} \sum_{i=1}^{n} E\left[\operatorname{Var}\left(X_{2, i} \mid X_{s, i}\right)\right] \leq \bar{\rho}_{2} \alpha_{2}+\bar{\alpha}_{2} .
$$

By symmetry, we only need to bound the following six mutual information constraints in (3) (the rest can be bounded using the same method, and the dimension super script ${ }^{(n)}$ is suppressed hereafter to simplify the notation):

$I\left(X_{1} X_{2} ; Y_{1} Y_{r} \mid X_{r}\right), I\left(X_{1} X_{2} X_{r} ; Y_{1} \mid X_{s}\right), I\left(X_{1} X_{r} ; Y_{1} \mid X_{2} X_{s}\right)$, $I\left(X_{1} X_{2} X_{r} ; Y_{1}\right), I\left(X_{1} X_{2} ; Y_{1} Y_{r} \mid X_{r} X_{s}\right), I\left(X_{1} ; Y_{1} Y_{r} \mid X_{2} X_{r} X_{s}\right)$.

Since

$$
\begin{aligned}
& \operatorname{Var}\left[Y_{1, i}\right]=1+\gamma_{11} \operatorname{Var}\left[X_{1, i}\right]+\gamma_{21} \operatorname{Var}\left[X_{2, i}\right]+\gamma_{r 1} \operatorname{Var}\left[X_{r, i}\right] \\
& \quad+2 \sqrt{\gamma_{11} \gamma_{21}} \operatorname{Cov}\left(X_{1, i}, X_{2, i}\right)+2 \sqrt{\gamma_{11} \gamma_{r 1}} \operatorname{Cov}\left(X_{1, i}, X_{r, i}\right) \\
& \quad+2 \sqrt{\gamma_{21} \gamma_{r 1}} \operatorname{Cov}\left(X_{2, i}, X_{r, i}\right),
\end{aligned}
$$

we can apply (25), (26), (28) and obtain

$$
\begin{aligned}
& \frac{1}{n} I\left(X_{1} X_{2} X_{r} ; Y_{1}\right)=\frac{1}{n} h\left(Y_{1}\right)-\frac{1}{2} \log (2 \pi e) \\
& \leq \frac{1}{2} \log \left(\frac{1}{n} \sum_{i=1}^{n} \operatorname{Var}\left[Y_{1, i}\right]\right) \\
& \leq \mathcal{C}\left(\gamma_{11}+\gamma_{21}+\gamma_{r 1}+2 \sqrt{\bar{\alpha}_{1} \gamma_{11} \gamma_{r 1}}+2 \sqrt{\bar{\alpha}_{2} \gamma_{21} \gamma_{r 1}}\right. \\
& \left.\quad+2 \sqrt{\gamma_{11} \gamma_{21}}\left(\sqrt{\rho_{1} \rho_{2} \alpha_{1} \alpha_{2}}+\sqrt{\bar{\alpha}_{1} \bar{\alpha}_{2}}\right)\right),
\end{aligned}
$$

with the first inequality obtained as follows

$$
\begin{aligned}
\frac{1}{n} h\left(Y_{1}\right) & \leq \frac{1}{n} \sum_{i=1}^{n} h\left(Y_{1, i}\right) \leq \frac{1}{2 n} \sum_{i=1}^{n} \log \left(2 \pi e \operatorname{Var}\left[Y_{1, i}\right]\right) \\
& \leq \frac{1}{2} \log \left(\frac{2 \pi e}{n} \sum_{i=1}^{n} \operatorname{Var}\left[Y_{1, i}\right]\right),
\end{aligned}
$$

where the second inequality is due to the maximum entropy lemma [35] and the last step follows from Jensen's inequality. Similarly, by the fact that

$$
\begin{aligned}
& \operatorname{Cov}\left(X_{1}, X_{r}\right)=E\left[\operatorname{Cov}\left(X_{1}, X_{r} \mid X_{s}\right)\right]+\operatorname{Cov}\left(E\left(X_{1} \mid X_{s}\right), E\left(X_{r}\right)\right) \\
& =E\left[\operatorname{Cov}\left(X_{1}, X_{r} \mid X_{s}\right)\right] \\
& \text { and } \\
& \begin{aligned}
E\left[\operatorname{Var}\left(Y_{1, i} \mid X_{s, i}\right)\right]=1+ & \gamma_{r 1} \operatorname{Var}\left(X_{r, i}\right)+\gamma_{11} E\left[\operatorname{Var}\left(X_{1, i} \mid X_{s, i}\right)\right] \\
+\gamma_{21} E\left[\operatorname{Var}\left(X_{2, i} \mid X_{s, i}\right)\right] & +2 \sqrt{\gamma_{11} \gamma_{21}} E\left[\operatorname{Cov}\left(X_{1, i}, X_{2, i} \mid X_{s, i}\right)\right] \\
& +2 \sqrt{\gamma_{11} \gamma_{r 1}} E\left[\operatorname{Cov}\left(X_{1, i}, X_{r, i} \mid X_{s, i}\right)\right] \\
& +2 \sqrt{\gamma_{21} \gamma_{r 1}} E\left[\operatorname{Cov}\left(X_{2, i}, X_{r, i} \mid X_{s, i}\right)\right]
\end{aligned}
\end{aligned}
$$


we can obtain

$$
\begin{aligned}
& \frac{1}{n} I\left(X_{1} X_{2} X_{r} ; Y_{1} \mid X_{s}\right)=\frac{1}{n} h\left(Y_{1} \mid X_{s}\right)-\frac{1}{2} \log (2 \pi e) \\
& \leq \frac{1}{2} \log \left(\frac{1}{n} \sum_{i=1}^{n} E \operatorname{Var}\left[Y_{1, i} \mid X_{s, i}\right]\right) \\
& \leq \mathcal{C}\left(\gamma_{11}\left(\bar{\alpha}_{1}+\bar{\rho}_{1} \alpha_{1}\right)+\gamma_{21}\left(\bar{\alpha}_{2}+\bar{\rho}_{2} \alpha_{2}\right)+\gamma_{r 1}+2 \sqrt{\gamma_{11} \gamma_{21} \bar{\alpha}_{1} \bar{\alpha}_{2}}\right. \\
& \left.\quad+2 \sqrt{\gamma_{11} \gamma_{r 1} \bar{\alpha}_{1}}+2 \sqrt{\gamma_{21} \gamma_{r 1} \bar{\alpha}_{2}}\right) .
\end{aligned}
$$

Similarly we can obtain

$$
\begin{aligned}
E\left[\operatorname{Var}\left(Y_{1, i} \mid X_{2, i} X_{s, i}\right)\right] & \\
=1 & +E\left[\operatorname{Var}\left(\sqrt{\gamma_{11}} X_{1, i}+\sqrt{\gamma_{r 1}} X_{r, i} \mid X_{2, i} X_{s, i}\right)\right] \\
=1 & +E\left[\operatorname{Var}\left(\sqrt{\gamma_{11}} X_{1, i}+\sqrt{\gamma_{r 1}} X_{r, i} \mid X_{r, i} X_{2, i} X_{s, i}\right)\right] \\
& +E\left[\operatorname{Var}\left(E\left(\sqrt{\gamma_{11}} X_{1, i}+\sqrt{\gamma_{r 1}} X_{r, i} \mid X_{r, i}\right) \mid X_{2, i} X_{s, i}\right)\right] \\
\leq 1 & +\gamma_{11}\left(E\left[\operatorname{Var}\left(X_{1, i} \mid X_{r, i} X_{s, i}\right)\right]+E\left[E^{2}\left(X_{1, i} \mid X_{r, i}\right)\right]\right) \\
& +\gamma_{r 1} E\left(X_{r, i}^{2}\right)+2 \sqrt{\gamma_{11} \gamma_{r 1}} E\left(X_{r, i} E\left(X_{1, i} \mid X_{r, i}\right)\right),
\end{aligned}
$$

where the second equality is due to Lemma 1 and the inequality is from (30). After applying the Cauchy-Schwarz inequality and power constraints, we can obtain

$$
\begin{aligned}
& \frac{1}{n} I\left(X_{1} X_{r} ; Y_{1} \mid X_{2} X_{s}\right) \leq \frac{1}{2} \log \left(\frac{1}{n} \sum_{i=1}^{n} E \operatorname{Var}\left[Y_{1, i} \mid X_{2 i} X_{s, i}\right]\right) \\
& \leq \mathcal{C}\left(\gamma_{11}\left(\bar{\rho}_{1} \alpha_{1}+\bar{\alpha}_{1}\right)+\gamma_{r 1}+2 \sqrt{\gamma_{11} \gamma_{r 1} \bar{\alpha}_{1}}\right) .
\end{aligned}
$$

Let $\boldsymbol{A}_{i}$ be the conditional covariance matrix of $\left(Y_{1, i}, Y_{r, i}\right)$ given $X_{r, i}$, then

$$
\begin{aligned}
& \left|\frac{1}{n} \sum_{i=1}^{n} \boldsymbol{A}_{i}\right|=1+\left(\gamma_{11}+\gamma_{1 r}\right) \frac{1}{n} \sum_{i=1}^{n} E\left[\operatorname{Var}\left(X_{1, i} \mid X_{r, i}\right)\right] \\
& +\frac{\left(\gamma_{21}+\gamma_{2 r}\right)}{n} \sum_{i=1}^{n} E\left[\operatorname{Var}\left(X_{2, i} \mid X_{r, i}\right)\right]+\left(\sqrt{\gamma_{11} \gamma_{2 r}}-\sqrt{\gamma_{21} \gamma_{1 r}}\right)^{2} \\
& \quad \times \frac{1}{n} \sum_{i=1}^{n} E\left[\operatorname{Var}\left(X_{1, i} \mid X_{r, i}\right)\right] \times \frac{1}{n} \sum_{j=1}^{n} E\left[\operatorname{Var}\left(X_{2, j} \mid X_{r, j}\right)\right] \\
& +2\left(\sqrt{\gamma_{11} \gamma_{21}}+\sqrt{\gamma_{1 r} \gamma_{2 r}}\right) \frac{1}{n} \sum_{i=1}^{n} E\left[\operatorname{Cov}\left(X_{1, i}, X_{2, i} \mid X_{r, i}\right)\right] \\
& -\left(\sqrt{\gamma_{11} \gamma_{2 r}}-\sqrt{\gamma_{21} \gamma_{1 r}}\right)^{2}\left(\frac{1}{n} \sum_{i=1}^{n} E\left[\operatorname{Cov}\left(X_{1, i}, X_{2, i} \mid X_{r, i}\right)\right]\right)^{2} \\
& \leq 1+\left(\gamma_{11}+\gamma_{1 r}\right) \alpha_{1}+2\left(\sqrt{\gamma_{11} \gamma_{21}}+\sqrt{\gamma_{1 r} \gamma_{2 r}}\right) \lambda_{1} \sqrt{\rho_{1} \rho_{2} \alpha_{1} \alpha_{2}} \\
& +\left(\gamma_{21}+\gamma_{2 r}\right) \alpha_{2}+\left(\sqrt{\gamma_{11} \gamma_{2 r}}-\sqrt{\gamma_{21} \gamma_{1 r}}\right)^{2} \alpha_{1} \alpha_{2}\left(1-\lambda_{1}^{2} \rho_{1} \rho_{2}\right),
\end{aligned}
$$

where $\lambda_{1} \in[0,1]$ is a maximization parameter defined by

$$
\lambda_{1}=\min \left\{1, \frac{\sqrt{\gamma_{11} \gamma_{21}}+\sqrt{\gamma_{1 r} \gamma_{2 r}}}{\left(\sqrt{\gamma_{11 \gamma_{2 r}}}-\sqrt{\gamma_{21} \gamma_{1 r}}\right)^{2} \sqrt{\rho_{1} \rho_{2} \alpha_{1} \alpha_{2}}}\right\},
$$

with $\lambda_{1}=1$ if $\alpha_{1} \alpha_{2} \rho_{1} \rho_{2}=0$. We can therefore bound the following mutual information terms

$$
\begin{aligned}
& \frac{1}{n} I\left(X_{1} X_{2} ; Y_{1} Y_{r} \mid X_{r}\right) \leq \frac{1}{2} \log \left(\left|\frac{1}{n} \sum_{i=1}^{n} \boldsymbol{A}_{i}\right|\right) \\
& \leq \mathcal{C}\left(\left(\gamma_{11}+\gamma_{1 r}\right) \alpha_{1}+\left(\sqrt{\gamma_{11} \gamma_{2 r}}-\sqrt{\gamma_{21} \gamma_{1 r}}\right)^{2} \alpha_{1} \alpha_{2}\left(1-\lambda_{1}^{2} \rho_{1} \rho_{2}\right)\right. \\
& \left.\quad+\left(\gamma_{21}+\gamma_{2 r}\right) \alpha_{2}+2\left(\sqrt{\gamma_{11} \gamma_{21}}+\sqrt{\gamma_{1 r} \gamma_{2 r}}\right) \lambda_{1} \sqrt{\rho_{1} \rho_{2} \alpha_{1} \alpha_{2}}\right) .
\end{aligned}
$$

Similarly, let $\boldsymbol{B}_{i}$ and $\boldsymbol{C}_{i}$ be the conditional covariance matrix of $\left(Y_{1, i}, Y_{r, i}\right)$ given $\left(X_{r, i}, X_{s, i}\right)$ and given $\left(X_{2, i}, X_{r, i}, X_{s, i}\right)$, respectively, we have

$$
\begin{aligned}
& \left|\frac{1}{n} \sum_{i=1}^{n} \boldsymbol{B}_{i}\right|=1+\left(\gamma_{11}+\gamma_{1 r}\right) \frac{1}{n} \sum_{i=1}^{n} E\left[\operatorname{Var}\left(X_{1, i} \mid X_{r, i} X_{s, i}\right)\right]+ \\
& \frac{\left(\gamma_{21}+\gamma_{2 r}\right)}{n} \sum_{i=1}^{n} E\left[\operatorname{Var}\left(X_{2, i} \mid X_{r, i} X_{s, i}\right)\right]+\left(\sqrt{\gamma_{11} \gamma_{2 r}}-\sqrt{\gamma_{21} \gamma_{1 r}}\right)^{2} \\
& \times \frac{1}{n} \sum_{i=1}^{n} E\left[\operatorname{Var}\left(X_{1, i} \mid X_{r, i} X_{s, i}\right)\right] \frac{1}{n} \sum_{j=1}^{n} E\left[\operatorname{Var}\left(X_{2, j} \mid X_{r, j} X_{s, j}\right)\right],
\end{aligned}
$$

and

$$
\left|\frac{1}{n} \sum_{i=1}^{n} \boldsymbol{C}_{i}\right|=1+\left(\gamma_{11}+\gamma_{1 r}\right) \frac{1}{n} \sum_{i=1}^{n} E\left[\operatorname{Var}\left(X_{1, i} \mid X_{r, i} X_{s, i}\right)\right] .
$$

We can therefore bound the following terms

$$
\begin{aligned}
\frac{1}{n} I\left(X_{1} X_{2} ; Y_{1} Y_{r} \mid X_{r} X_{s}\right) & \leq \frac{1}{2} \log \left(\left|\frac{1}{n} \sum_{i=1}^{n} \boldsymbol{B}_{i}\right|\right) \\
\leq \mathcal{C}\left(\left(\gamma_{11}+\gamma_{1 r}\right) \bar{\rho}_{1} \alpha_{1}+\right. & \left(\gamma_{21}+\gamma_{2 r}\right) \bar{\rho}_{2} \alpha_{2} \\
+ & \left.\left(\sqrt{\gamma_{11} \gamma_{2 r}}-\sqrt{\gamma_{21} \gamma_{1 r}}\right)^{2} \bar{\rho}_{1} \bar{\rho}_{2} \alpha_{1} \alpha_{2}\right) \\
\frac{1}{n} I\left(X_{1} ; Y_{1} Y_{r} \mid X_{2} X_{r} X_{s}\right) & \leq \frac{1}{2} \log \left(\left|\frac{1}{n} \sum_{i=1}^{n} \boldsymbol{C}_{i}\right|\right) \\
& \leq \mathcal{C}\left(\left(\gamma_{11}+\gamma_{1 r}\right) \bar{\rho}_{1} \alpha_{1}\right)
\end{aligned}
$$

Using the similar procedures as we demonstrated above, we may bound the remaining inequalities in (3), and combining all the results and let $n \rightarrow \infty$, we obtain (4).

\section{APPENDIX B}

\section{ProOF OF PROPOSITION 3}

As in Appendix A, we first introduce auxiliary random variables $\alpha_{1}, \alpha_{2}, \rho_{1} \in[0,1]$ such that

$$
\begin{aligned}
\bar{\alpha}_{1} & =\frac{1}{n} \sum_{i=1}^{n} E\left[E^{2}\left(X_{1, i} \mid X_{r, i}\right)\right] \\
\bar{\alpha}_{2} & =\frac{1}{n} \sum_{i=1}^{n} E\left[E^{2}\left(X_{2, i} \mid X_{r, i}\right)\right], \\
\bar{\rho}_{1} \alpha_{1} & =\frac{1}{n} \sum_{i=1}^{n} E\left[\operatorname{Var}\left(X_{1, i} \mid X_{r, i} X_{s, i}\right)\right],
\end{aligned}
$$

we can thus obtain (20)-(22), (24)-(26) and (29) as in Appendix A. Since

$$
\begin{aligned}
& \frac{1}{n} \sum_{i=1}^{n} E\left[\operatorname{Cov}\left(X_{1, i}, X_{2, i} \mid X_{r, i}\right)\right] \\
& \leq \sqrt{\frac{1}{n} \sum_{i=1}^{n} E\left[\operatorname{Var}\left(X_{1, i} \mid X_{r, i}\right)\right] \frac{1}{n} \sum_{j=1}^{n} E\left[\operatorname{Var}\left(X_{2, j} \mid X_{r, j}\right)\right]} \\
& \leq \sqrt{\alpha_{1} \alpha_{2}},
\end{aligned}
$$

we can introduce an auxiliary variable $\rho \in[0,1]$ such that

$$
\frac{1}{n} \sum_{i=1}^{n} E\left[\operatorname{Cov}\left(X_{1, i}, X_{2, i} \mid X_{r, i}\right)\right]=\rho \sqrt{\alpha_{1} \alpha_{2}} \text {. }
$$


On the other hand, we can obtain from (27) that

$$
\begin{aligned}
& \frac{1}{n} \sum_{i=1}^{n} E\left[\operatorname{Cov}\left(X_{1, i}, X_{2, i} \mid X_{r, i}\right)\right] \\
& \leq \sqrt{\frac{1}{n} \sum_{i=1}^{n} E\left[\operatorname{Var}\left(E\left(X_{1, i} \mid X_{s, i}\right) \mid X_{r, i}\right)\right]} \\
& \times \sqrt{\frac{1}{n} \sum_{i=1}^{n} E\left[\operatorname{Var}\left(E\left(X_{2, i} \mid X_{s, i}\right) \mid X_{r, i}\right)\right]}
\end{aligned}
$$

which leads to the following observations

$$
\begin{gathered}
\alpha_{1} \rho^{2} \leq \frac{1}{n} \sum_{i=1}^{n} E\left[\operatorname{Var}\left(E\left(X_{1, i} \mid X_{s, i}\right) \mid X_{r, i}\right)\right] \leq \rho_{1} \alpha_{1}, \\
\alpha_{2} \rho^{2} / \rho_{1} \leq \frac{1}{n} \sum_{i=1}^{n} E\left[\operatorname{Var}\left(E\left(X_{2, i} \mid X_{s, i}\right) \mid X_{r, i}\right)\right] \leq \alpha_{2}, \\
\frac{1}{n} \sum_{i=1}^{n} E\left[\operatorname{Var}\left(X_{2, i} \mid X_{r, i} X_{s, i}\right)\right] \leq\left(1-\rho^{2} / \rho_{1}\right) \alpha_{2},
\end{gathered}
$$

where $\rho^{2} / \rho_{1}=0$ when $\rho=\rho_{1}=0$.

Now, by following the same procedure as in Appendix A, replacing $\rho_{2}$ by $\rho^{2} / \rho_{1}$, and bounding

$\left|\frac{1}{n} \sum_{i=1}^{n} \boldsymbol{A}_{i}\right| \leq 1+\left(\sqrt{\gamma_{11} \gamma_{2 r}}-\sqrt{\gamma_{21} \gamma_{1 r}}\right)^{2} \alpha_{1} \alpha_{2}\left(1-\rho^{2}\right)+$
$\left(\gamma_{11}+\gamma_{1 r}\right) \alpha_{1}+\left(\gamma_{21}+\gamma_{2 r}\right) \alpha_{2}+2\left(\sqrt{\gamma_{11} \gamma_{21}}+\sqrt{\gamma_{1 r} \gamma_{2 r}}\right) \rho \sqrt{\alpha_{1} \alpha_{2}}$,

we can obtain (5).

\section{REFERENCES}

[1] F. M. J. Willems, "The discrete memoryless multiple-access channel with partially cooperating encoders," IEEE Trans. Inf. Theory, vol. 29, pp. 441445, May 1983.

[2] S. I. Bross, A. Lapidoth, and M. A. Wigger, "The Gaussian MAC with conferencing encoders," in Proc. of IEEE ISIT, Jul. 2008.

[3] O. Simeone, D. Gündüz, H. V. Poor, A. J. Goldsmith, and S. Shamai, "Compound multiple-access channels with partial cooperation," IEEE Trans. Inf. Theory, vol. 55, pp. 2425-2441, Jun. 2009.

[4] I. Marić, R. Yates, and G. Kramer, "Capacity of interference channels with partial transmitter cooperation," IEEE Trans. Inf. Theory, vol. 53, pp. 3536-3548, Oct. 2007.

[5] N. Devroye, P. Mitran, and V. Tarokh, "Achievable rates in cognitive radio channels," IEEE Trans. Inf. Theory, vol. 52, pp. 1813-1827, May 2006.

[6] I.-H. Wang and D. N. C. Tse, "Interference mitigation through limited transmitter cooperation," IEEE Trans. Inf. Theory, vol. 57, pp. 2941-2965, May 2011.

[7] V. M. Prabhakaran and P. Viswanath, "Interference channels with source cooperation," IEEE Trans. Inf. Theory, vol. 57, pp. 156-186, Jan. 2011.

[8] A. Sendonaris, E. Erkip, and B. Aazhang, "User cooperation diversityPart I: System description," IEEE Trans. Communications, vol. 51, pp. 1927-1938, Nov. 2003.

[9] A. Sendonaris, E. Erkip, and B. Aazhang, "User cooperation diversityPart II: Implementation aspects and performance analysis," IEEE Trans. Communications, vol. 51, pp. 1939-1948, Nov. 2003.

[10] J. N. Laneman, D. N. C. Tse, and G. W. Wornell, "Cooperative diversity in wireless networks: efficient protocols and outage behavior," IEEE Trans. Inf. Theory, vol. 50, pp. 3062-3080, Dec. 2004.

[11] A. Høst-Madsen, "Capacity bounds for cooperative diversity," IEEE Trans. Inf. Theory, vol. 52, pp. 1522-1544, Apr. 2006.

[12] C. T. K Ng, N. Jindal, A. J. Goldsmith, and U. Mitra, "Capacity gain from two-transmitter and two-receiver cooperation," IEEE Trans. Inf. Theory, vol. 53, pp. 3822-3827, Oct. 2007.

[13] S. Ray, Energy Efficient Multiple Antenna Communication, Ph.D. thesis, MIT, Sep. 2006.
[14] R. Zakhour and D. Gesbert, "Optimized data sharing in multicell MIMO with finite backhaul capacity," IEEE Trans. Signal Processing, vol. 59, pp. 6102-6111, Dec. 2011.

[15] M. Wiese, H. Boche, I. Bjelaković, and V. Jungnickel, "The compound multiple access channel with partially cooperating encoders," IEEE Trans. Inf. Theory, vol. 57, pp. 3045-3066, May 2011.

[16] T. M. Cover and A. El Gamal, "Capacity theorems for the relay channel," IEEE Trans. Inf. Theory, vol. 25, pp. 572-584, Sep. 1979.

[17] A. Høst-Madsen and J. Zhang, "Capacity bounds and power allocation for wireless relay channels," IEEE Trans. Inf. Theory, vol. 51, pp. 2020 2040, Jun. 2005.

[18] G. Kramer and A. J. van Wijngaarden, "On the white Gaussian multipleaccess relay channel," in Proc. of IEEE ISIT, Jan. 2000.

[19] G. Kramer, M. Gastpar and P. Gupta, "Cooperative strategies and capacity theorems for relay networks," IEEE Trans. Inf. Theory, vol. 51, pp. 3037-3063, Sep. 2005

[20] Y. Liang and G. Kramer, "Rate regions for relay broadcast channels," IEEE Trans. Inf. Theory, vol. 53, pp. 3517-3535, Oct. 2007.

[21] O. Sahin and E. Erkip, "Achievable rates for the Gaussian interference relay channel," in Proc. of IEEE GLOBECOM, Nov. 2007.

[22] Y. Tian and A. Yener, "The Gaussian interference relay channel: improved achievable rates and sum rate upperbounds using a potent relay," IEEE Trans. Inf. Theory, vol. 57, pp. 2865-2879, May 2011.

[23] A. Avestimehr and T. Ho, "Approximate capacity of the symmetric halfduplex Gaussian butterfly network," in Proc. of IEEE ITW, Jun. 2009.

[24] D. Gündüz, O. Simeone, A. J. Goldsmith, H. V. Poor, and S. Shamai, "Multiple multicasts with the help of a relay," IEEE Trans. Inf. Theory, vol. 56, pp. 6142-6158, Dec. 2010.

[25] L. Zhou and W. Yu, "Incremental relaying for the Gaussian interference channel with a degraded broadcasting relay," in Proc. of Allerton, Sep. 2011.

[26] S.R. Li, R.W. Yeung and N. Cai, "Linear network coding," IEEE Trans. Inf. Theory, vol. 49, No. 2, pp. 371-381, Feb. 2003.

[27] R. Koetter and M. Médard, "An algebraic approach to network coding," IEEE Trans. Networking, vol. 11, pp. 782-795, Oct. 2003.

[28] S. Katti, H. Rahul, W. Hu, D. Katabi, M. Médard, and J. Crowcroft, "XORs in the air: practical wireless network coding," IEEE/ACM Trans. Networking, vol. 16, pp. 497-510, Jun. 2008

[29] S. Katti, I. Marić, A. J. Goldsmith, D. Katabi, and M. Médard, "Joint relaying and network coding in wireless networks," in Proc. of IEEE ISIT, Jun. 2007.

[30] I. Marić, A. J. Goldsmith, and M. Médard, "Multihop analog network coding via amplify-and-forward: the high SNR regime," IEEE Trans. Inf. Theory, vol. 58, pp. 793-803, Feb. 2012.

[31] S. H. Lim, Y.-H. Kim, A. El Gamal and S.-Y. Chung, "Noisy network coding," IEEE Trans. Inf. Theory, vol. 57, No. 5, pp. 3132-3152, May 2011.

[32] J. Du, M. Xiao, and M. Skoglund, "Cooperative network coding strategies for wireless relay networks with backhaul," IEEE Trans. Communications, vol. 59, pp. 2502-2514, Sep. 2011.

[33] J. Du, M. Xiao, and M. Skoglund, "Capacity bounds for backhaulsupported wireless multicast relay networks with cross-links," in Proc. of IEEE ICC, Jun. 2011.

[34] R. Koetter, M. Effros, and M. Médard, "A theory of network equivalence-part I: point-to-point channels," IEEE Trans. Inf. Theory, vol. 57, No. 2, pp. 972-995, Feb. 2011.

[35] T. M. Cover and J. A. Thomas, Elements of Information Theory, New York, Wiley, 2006.

[36] A. B. Carleial, "Interference channels," IEEE Trans. Inf. Theory, vol. 24, pp. 60-70, Jan. 1978.

[37] B. Rimoldi and R. Urbanke, "A rate-splitting approach to the Gaussian multiple-access channel," IEEE Trans. Inf. Theory, vol. 42, pp. 364-375, Mar. 1996.

[38] A. B. Carleial, "Multiple-access channels with different generalized feedback signals," IEEE Trans. Inf. Theory, vol. 28, pp. 841-850, Nov. 1982.

[39] F. M. J. Willems, Information Theoretical Results for the Discrete Memoryless Multiple Access Channel, Ph.D. thesis, Katholieke Univ. Leuven, Oct. 1982

[40] R. Guseo, "Partial and ecological correlation: a common three-term covariance decomposition," Statistical Methods and Applications, vol. 19, pp. 31-46, Mar. 2010. 


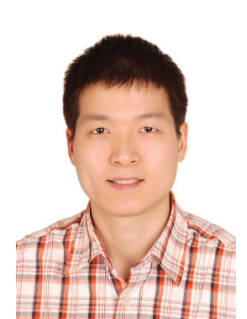

Jinfeng Du (S'07) received his B.Eng. degree in electronic information engineering from the University of Science and Technology of China (USTC), Hefei, China in 2004, M.Sc. degree in electronic engineering and Tekn. Lic. degree in electronics and computer systems, both from the Royal Institute of Technology (KTH), Stockholm, Sweden, in 2006 and 2008 , respectively. He is currently a Ph.D. student in telecommunications at KTH. He received the best paper award from IC-WCSP in October 2010, the "Hans Werthén Grant" from the Royal Swedish Academy of Engineering Science (IVA) in March 2011, the grant from Ericsson Research Foundation in May 2011, and the "Chinese Government Award for Outstanding Self-Financed Students Abroad" in May 2012.

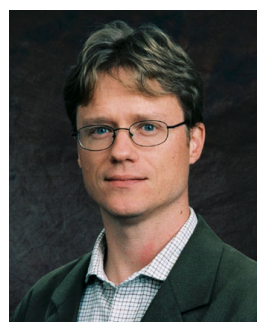

Mikael Skoglund (S'93-M'97-SM'04) received the Ph.D. degree in 1997 from Chalmers University of Technology, Sweden. In 1997, he joined the Royal Institute of Technology (KTH), Stockholm, Sweden, where he was appointed to the Chair in Communication Theory in 2003. At KTH, he heads the Communication Theory Lab and he is the Assistant Dean for Electrical Engineering.

Dr. Skoglund's research interests are in the theoretical aspects of wireless communications. $\mathrm{He}$ has worked on problems in source-channel coding, coding and transmission for wireless communications, Shannon theory and statistical signal processing. He has authored and co-authored more than 300 scientific papers in these areas, and he holds six patents.

Dr. Skoglund has served on numerous technical program committees for IEEE conferences. During 2003-08 he was an associate editor with the IEEE Transactions on Communications and he is presently on the editorial board for IEEE Transactions on Information Theory.

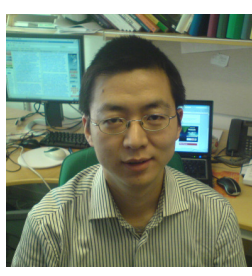

Ming Xiao (S'2002-M'2007) was born in the SiChuan Province, P. R. China, in 1975. He received Bachelor and Master degrees in Engineering from the University of Electronic Science and Technology of China, ChengDu in 1997 and 2002, respectively. He received Ph.D degree from Chalmers University of technology, Sweden in November 2007. From 1997 to 1999 , he worked as a network and software engineer in ChinaTelecom. From 2000 to 2002, he also held a position in the SiChuan communications administration. From November 2007 to now, he has been in ACCESS Linnaeus center, school of electrical engineering, Royal Institute of Technology, Sweden, where he is currently an assistant professor. He received the best paper Awards in "IC-WCSP" (International Conference on Wireless Communications and Signal Processing) in 2010 and "IEEE ICCCN" (International Conference on Computer Communication Networks) in 2011. Dr. Xiao received "Chinese Government Award for Outstanding SelfFinanced Students Studying Abroad" in March, 2007. He got "Hans Werthén Grant" from royal Swedish academy of engineering science (IVA) in March 2006. He received "Ericsson Research Funding" from Ericsson in 2010.

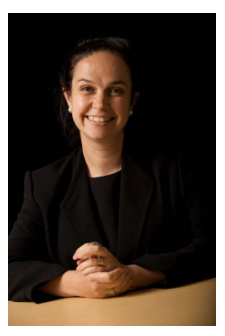

Muriel Médard is a Professor of Electrical Engineering at MIT. She was previously an Assistant Professor in the ECE Department at UIUC and a Staff Member at MIT Lincoln Laboratory. She received B.S. degrees in EECS, in Mathematics, and in Humanities, as well as M.S. and Sc D. degrees in EE, all from MIT. She has served as an Associate Editor for the Optical Communications and Networking Series of the IEEE Journal on Selected Areas in Communications, the IEEE Transactions on Information Theory and the OSA Journal of Optical Networking. She has served as a Guest Editor for the IEEE Journal of Lightwave Technology, the IEEE Transactions on Information Theory (twice), the IEEE Journal on Selected Areas in Communications and the IEEE Transactions on Information Forensic and Security. She serves as an associate editor for the IEEE/OSA Journal of Lightwave Technology. She is a member of the Board of Governors of the IEEE Information Theory Society and currently serves as the President. She has served as TPC co-chair of ISIT, WiOpt and CONEXT. She was awarded the 2009 IEEE Communication Society and Information Theory Society Joint Paper Award, the 2009 IEEE William R. Bennett Prize in the Field of Communications, and the 2002 IEEE Leon K. Kirchmayer Prize Paper Award. She was co-winner of the 2004 MIT Harold E. Edgerton Faculty Achievement Award. In 2007, she was named a Gilbreth Lecturer by the National Academy of Engineering. Professor Médard's research interests are in the areas of network coding and reliable communications, particularly for optical and wireless networks. 